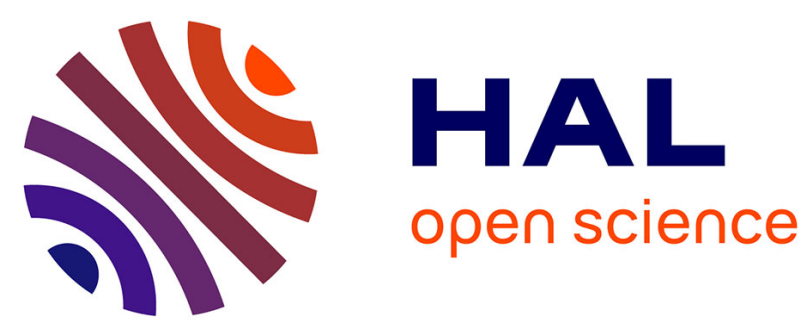

\title{
The Halfspace Matching Method: a new method to solve scattering problem in infinite media
} Anne-Sophie Bonnet-Ben Dhia, Sonia Fliss, Antoine Tonnoir

\section{To cite this version:}

Anne-Sophie Bonnet-Ben Dhia, Sonia Fliss, Antoine Tonnoir. The Halfspace Matching Method: a new method to solve scattering problem in infinite media. Journal of Computational and Applied Mathematics, 2018, 338, pp.44-68. 10.1016/j.cam.2018.01.021 . hal-01561339v3

\section{HAL Id: hal-01561339 \\ https://hal.science/hal-01561339v3}

Submitted on 22 Nov 2018

HAL is a multi-disciplinary open access archive for the deposit and dissemination of scientific research documents, whether they are published or not. The documents may come from teaching and research institutions in France or abroad, or from public or private research centers.
L'archive ouverte pluridisciplinaire HAL, est destinée au dépôt et à la diffusion de documents scientifiques de niveau recherche, publiés ou non, émanant des établissements d'enseignement et de recherche français ou étrangers, des laboratoires publics ou privés. 


\title{
The Halfspace Matching Method : a new method to solve scattering problems in infinite media
}

\author{
ANNE-SOPHIE BONNET-BEN DHIA ${ }^{a}$, SONIA FLISS ${ }^{a, *}$, ANTOINE \\ TONNOIR $^{b}$ \\ ${ }^{a}$ POEMS (UMR 7231 CNRS-ENSTA-INRIA), Universit Paris Saclay \\ ENSTA, 828 Boulevard des Marechaux, 91762 Palaiseau Cedex, France \\ ${ }^{b}$ Normandie University, INSA Rouen Normandie, LMI, 76000 Rouen, France
}

\begin{abstract}
We are interested in acoustic wave propagation in time harmonic regime in a two-dimensional medium which is a local perturbation of an infinite isotropic or anisotropic homogeneous medium. We investigate the question of finding artificial boundary conditions to reduce the numerical computations to a neighborhood of this perturbation. Our objective is to derive a method which can extend to the anisotropic elastic problem for which classical approaches fail. The idea consists in coupling several semi-analytical representations of the solution in halfspaces surrounding the defect with a Finite Element computation of the solution around the defect. As representations of the same function, they have to match in the infinite intersections of the halfspaces. It leads to a formulation which couples, via integral operators, the solution in a bounded domain including the defect and its traces on the edge of the halfspaces. A stability property is shown for this new formulation.
\end{abstract}

Keywords: Anisotropic Helmholtz equation; plane-waves representations; Fourier Transform; Integral operators; Domain Decomposition Methods.

\section{Introduction and model problem}

This work is motivated by the numerical simulation of Non Destructive Testing or Structural Health Monitoring experiments in anisotropic elastic media (see for instance (4)). More precisely, we are interested in simulating the

*sonia.fliss@ensta-paristech.fr 
diffraction of time-harmonic waves by a localized perturbation in a homogeneous two dimensional infinite anisotropic elastic medium. Since the medium is infinite, there are theoretical difficulties -how to define the so called outgoing solution of such problem?- and numerical difficulties -can we introduce an equivalent formulation which is suitable for numerical purposes (for instance a formulation set in a bounded domain with appropriate boundary conditions)?

This is an old problematic (15) for time harmonic scalar wave equations and there exist several methods. They are all based on the natural idea of reducing the pure numerical computations to a bounded domain containing the perturbations (achieved using for instance Finite Element methods). A first class of methods consists in applying an artificial boundary conditions, around the bounded domain, which is transparent or approximately transparent as in : (1) integral equation techniques, (2) Dirichlet-to-Neumann approaches providing that the boundary is properly chosen to allow separation of variables and (3) local radiation conditions at finite distance constructed as local approximations at various order of the exact non local condition. These techniques were first introduced for the time harmonic scalar wave equation - the Helmholtz equation - and then extended to isotropic elasticity problems using simply the Helmholtz decomposition of the displacement field in terms of potential (see for instance (16)). However it seems that all these techniques either do not extend to anisotropic elastic media - the separation of variables is not possible anymore to determine the Dirichlet-to-Neumann (DtN) operator - or do extend but with a tremendous computational cost for the integral equation techniques, the Green tensor depends not only on the distance between two points but also on the orientation (26). A second class of methods consists in surrounding the computational domain by a Perfectly Matched absorbing Layer (PML). PML techniques are very popular because they are efficient and easy to implement in a large class of problems. But they may be inoperant. Roughly speaking, the PML absorbs the wave with an outgoing phase velocity, preventing them to come back in the computational domain, while in order to catch the physical solution, it should absorb the waves with outgoing group velocities. That is why to our knowledge the standard PML technique works for isotropic elastic media (in which the waves with outgoing phase velocities have outgoing group velocities and vice versa) but cannot work for general anisotropic elastic media where the two velocities may differ $(3 ; 23 ; 6)$. Let us mention finally the pole condition 
method which was developped recently and adapted to anisotropic elastic waveguide in the past few years $(19 ; 18)$.

By contrast, our method which is inspired by the method developped in $(12 ; 13)$ for locally perturbed periodic media can cover all the cases. It is based on a simple idea: the solution of homogeneous -isotropic or anisotropic, acoustic or elastic- halfspace problems can be expressed thanks to its trace on the halfspace boundary. As several halfspaces surrounding the perturbations are needed to recover the whole domain, they will necessarily overlap. The second step is then to find conditions to ensure the compatibility of the representations in the overlapping zones. This method has links with domain decomposition methods with overlap $(22 ; 11 ; 25)$, with the specific difficulty that here the overlapping zones are unbounded. More precisely, the idea is to split the whole domain into five parts:

- a square that includes the defect (and all the inhomogeneities) in which we will use a Finite Element representation of the solution,

- and 4 half-planes, parallel to the four edges of the square in which the medium is homogeneous.

Taking advantage of the homogeneity of the medium in a half-plane, we can give an explicit (integral) expression of the solution given (for instance) its trace on the edge of the half-plane, via the Fourier transform in the transverse direction. With these integral representations and the Finite Element representation of the solution in the square, we can formulate a coupled problem. To ensure the compatibility of the different representations, as in domain decomposition methods, we impose transmission conditions on the edges of the subdomains. This leads us to a system of coupled equations where the unknowns are the solution in the bounded square and the traces of the solution on the edges of the half-planes.

Obviously, compared to absorbing layers methods, this approach is more costly due to the additional unknowns (the traces) linked by non-local integral equations. One counterpart is that this additional computation of the traces enables to reconstruct a posteriori the solution in the half-planes (and therefore in the whole domain), which is impossible for instance when using non exact absorbing boundary conditions or PML. 
In the paper, we consider the simple model case of a scalar equation: the dissipative anisotropic Helmholtz equation. We reformulate the diffraction problem and analyse the properties of this reformulation. Let us underline that, thought our analysis holds only for dissipative media, the method gives good numerical results also in the non-dissipative case. We will explain, in a dedicated section, the theoretical difficulties raised by the case without dissipation. We should also emphasize that this approach remains valid for anisotropic elastic media since it mainly relies on the homogeneity of the medium in the half-planes to get the Fourier representations (see (24)). The complete description of the method in the elastic case will be the topic of another paper.

The general model problem that we consider in this paper is then

$$
\mid-\operatorname{div}(A(x, y) \nabla p)-\omega_{\varepsilon}^{2} \rho(x, y) p=f \quad \text { in } \quad \Omega,
$$

in the time harmonic regime at the frequency $\operatorname{Re}\left(\omega_{\varepsilon}\right)=\omega$ with a small absorption $\operatorname{Im}\left(\omega_{\varepsilon}\right)=\varepsilon>0$, where $A$ is a symmetric positive definite matrix of $\left(L^{\infty}(\Omega)\right)^{2 \times 2}$ modelling the anisotropy and $\rho$ is a strictly positive function of $L^{\infty}(\Omega)$.

The propagation domain $\Omega$ is typically $\mathbb{R}^{2}$, or $\mathbb{R}^{2}$ minus a set of obstacles which are included in a bounded region

$$
\exists a>0, \quad \partial \Omega \subset \Omega_{a} \equiv(-a, a)^{2} .
$$

In presence of obstacles, some boundary conditions have to be added to the model. The source term $f$ is supposed to be a function of $L^{2}(\Omega)$ with a compact support included in $\Omega_{a}$. Finally, the matrix $A$ is a local perturbation of a constant matrix $A_{0}$

$$
\operatorname{supp}\left(A-A_{0}\right) \subset \Omega_{a}, \text { where } A_{0}=\left(\begin{array}{ll}
c_{1} & c_{3} \\
c_{3} & c_{2}
\end{array}\right) \text { with }\left\{\begin{array}{l}
c_{1}, c_{2}>0 \\
c_{1} c_{2}-\left(c_{3}\right)^{2}>0
\end{array}\right.
$$

and the function $\rho$ is a local perturbation of a constant function, which is taken, without loss of generalities, equal to 1

$$
\operatorname{supp}(\rho-1) \subset \Omega_{a} .
$$


For variational boundary conditions on $\partial \Omega$-for instance Neumann or Dirichlet conditions- it is well known that thanks to the dissipation, this problem admits a unique solution in $H^{1}(\Omega)$.

To clarify the presentation of the method, we will consider three situations of increasing difficulty.

(Case 1) The propagation medium is here $\Omega=\mathbb{R}^{2} \backslash \Omega_{a}$ and non homogeneneous Dirichlet boundary conditions are imposed on its boundary. The source term $f=0, A=A_{0}=I d$ and $\rho=1$ in $\Omega$. Coupling analytical representations of the solution in the 4 halfspaces surrounding the obstacle $\mathcal{O}=\Omega_{a}$ and ensuring that all representations match, we end up with a system of integral equations whose unknowns are the 4 traces of the solution on the edges of the halfspaces. We show stability and well-posedness of this formulation in a framework which is suitable for numerical simulations. Finally, we show in Appendix A that $\mathcal{O}$ can be a triangle (resp. any other convex polygon with $\mathrm{N}$ sides) and a similar approach can be applied but 3 unknowns (resp. N unknowns) are involved.

(Case 2) We consider here the general isotropic case $A_{0}=I d$, in presence of source terms and possible perturbations of the geometry, the matrix $A$ and the coefficient $\rho$. The idea here is that any perturbation can be taken into account using a Finite Element (FE) method, as soon as it is contained in a bounded region. Here, we examine the coupling between the FE representation of the solution and the system of integral equations obtained in the previous case. We point out the importance of the presence of an overlap between the FE box and each halfspace. Thanks to this overlap, we show again stability properties and wellposedness for this problem.

(Case 3) We consider here the general anisotropic case. We investigate the influence of the anisotropy on the extension of the previous results.

We give in the next section the main results of the paper. More precisely, we explain formally how to derive the formulations corresponding to each situation and give the associated stability results. Moreover, we show numerical results to illustrate the efficiency of the method and to motivate the reader to continue the next sections which are much more technical. Sections 3, 4 and 
5 correspond to the theoretical study of, respectively the Case 1, the Case 2 and the Case 3 and contain the proofs of the associated theorems, respectively Theorem 2.2, Theorem 2.5 and Theorem 2.6. In Section 7, we explain the theoretical difficulties of the non dissipative case $(\varepsilon=0)$. Even if there are still some open questions in that case, we obtain convincing numerical results when extending formally the halfspace matching method.

Remark 1.1. As explained before, this method was inpired by previous works where the medium is periodic in two directions outside a bounded region(12; 13). Let us emphasize that in these works, no stability results on the method was obtained. What we claim is that thanks to the results of the present paper, we could now obtain stability result of the formulation in the periodic case and perform the numerical analysis. This will be the topic of another paper.

\section{Main results}

\subsection{Case 1 : the halfspace matching method for the isotropic case}

We consider the following problem: let $\Omega=\mathbb{R}^{2} \backslash \Omega_{a}, g \in H^{1 / 2}(\partial \Omega)$ and find the unique solution $p \in H^{1}(\Omega)$ of

$$
\mid \begin{array}{llr}
-\Delta p-\omega_{\varepsilon}^{2} p=0 & \text { in } & \Omega \\
p=g & \text { on } & \partial \Omega
\end{array}
$$

The domain $\Omega$ is the union of 4 half-planes $\Omega_{a}^{j}$ that lie on the 4 edges of the square $\Omega_{a}$. Using the following local coordinates for all $j \in \llbracket 0,3 \rrbracket$

$$
\left[\begin{array}{l}
x^{j} \\
y^{j}
\end{array}\right]=\left[\begin{array}{rr}
\cos \left(\theta_{j}\right) & -\sin \left(\theta_{j}\right) \\
\sin \left(\theta_{j}\right) & \cos \left(\theta_{j}\right)
\end{array}\right]\left[\begin{array}{l}
x \\
y
\end{array}\right], \quad \text { where } \theta_{j}=\frac{j \pi}{2} .
$$

the half-planes are defined as follows for all $j \in \llbracket 0,3 \rrbracket$

$$
\Omega_{a}^{j}=\left\{x^{j} \geq a\right\} \times\left\{y^{j} \in \mathbb{R}\right\} \quad \Sigma_{a}^{j} \equiv \partial \Omega_{a}^{j}:=\left\{x^{j}=a\right\} \times\left\{y^{j} \in \mathbb{R}\right\} .
$$

Finally, we denote

$$
\Sigma_{a a}=\partial \Omega_{a} \quad \text { and } \quad \Sigma_{a a}^{j}=\Sigma_{a a} \cap \Sigma_{a}^{j} .
$$

These notations are summarized on Figure 1. 


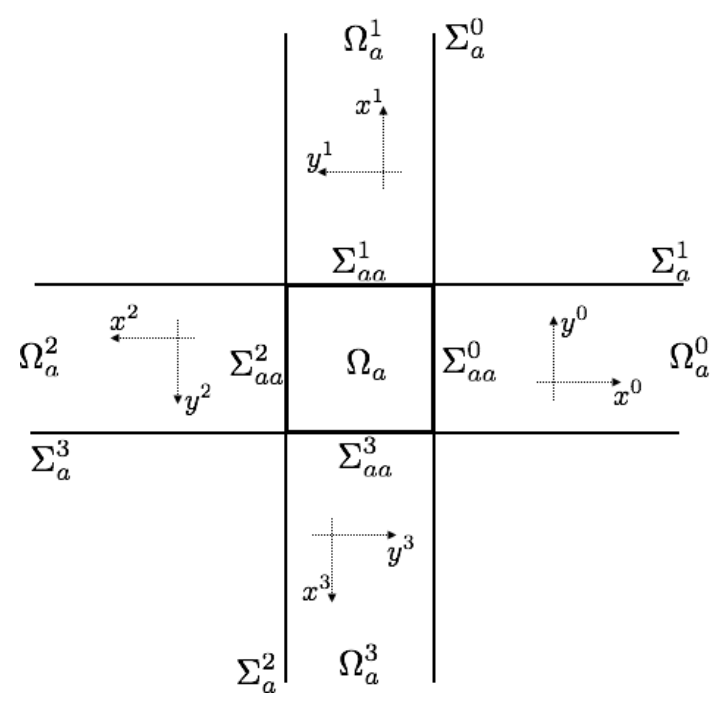

Figure 1: The notations defined in (5-6-7).

As explained in the introduction, the formulation uses the representation of the solution in each halfspace $\Omega_{a}^{j}$ surrounding the obstacle. Let us then introduce the halfspace problem: for any $\psi \in H^{1 / 2}\left(\Sigma_{a}^{j}\right)$, find the unique solution $P^{j}(\psi) \in H^{1}\left(\Omega_{a}^{j}\right)$

$$
\mid \begin{array}{ll}
-\Delta P^{j}-\omega_{\varepsilon}^{2} P^{j}=0 & \text { in } \quad \Omega_{a}^{j} \\
P^{j}=\psi & \text { on } \quad \Sigma_{a}^{j}
\end{array}
$$

Using the Fourier transform in the $y^{j}$-direction, it is easy to see that, the solution of (8) is given by

$\forall\left(x^{j}, y^{j}\right) \in[a,+\infty[\times \mathbb{R}$

$$
P^{j}(\psi)\left(x^{j}, y^{j}\right)=\frac{1}{\sqrt{2 \pi}} \int_{\mathbb{R}} \widehat{\psi}(\xi) e^{\imath \sqrt{\omega_{\varepsilon}^{2}-\xi^{2}}\left(x^{j}-a\right)} e^{\imath \xi y^{j}} d \xi
$$

where the square root is defined with the convention $\operatorname{Im} \sqrt{ } \geq 0$ and $\widehat{\psi}$ is the Fourier transformation of $\psi$ using the convention,

$$
\forall \xi \in \mathbb{R}, \quad \widehat{\psi}(\xi)=\frac{1}{\sqrt{2 \pi}} \int_{\mathbb{R}} \psi\left(y^{j}\right) e^{-\imath \xi y^{j}} d y^{j} .
$$


Let us now derive the halfspace matching formulation which involves only the traces of the solution $p$ on the edges $\Sigma_{a}^{j}$ of $\Omega_{a}^{j}$ that we denote

$$
\varphi^{j}=\left.p\right|_{\Sigma_{a}^{j}}
$$

We have thanks to the boundary conditions satisfied by $p$ in (4)

$$
\left.\varphi^{j}\right|_{\Sigma_{a a}^{j}}=\left.g\right|_{\Sigma_{a a}^{j}} .
$$

Moreover, because $p$ is solution of (4), its restriction to $\Omega_{a}^{j}$ is solution of (8) with $\psi=\varphi^{j}$ so

$$
\left.p\right|_{\Omega_{a}^{j}}=P^{j}\left(\varphi^{j}\right) .
$$

The trace of $p$ on $\Omega_{a}^{0} \cap \Sigma_{a}^{1}$ is given by $\varphi^{1}$ by (10) and by $P^{0}\left(\varphi^{0}\right)$ by (12) so we have necessarily

$$
\left.\varphi^{1}\right|_{\Sigma_{a}^{1} \cap \Omega_{a}^{0}}=\left.P^{0}\left(\varphi^{0}\right)\right|_{\Sigma_{a}^{1} \cap \Omega_{a}^{0}} .
$$

Applying this reasoning to the other half lines $\Sigma_{a}^{j \pm 1} \cap \Omega_{a}^{j}$, we get

$$
\left.\varphi^{j \pm 1}\right|_{\Sigma_{a}^{j \pm 1} \cap \Omega_{a}^{j}}=\left.P^{j}\left(\varphi^{j}\right)\right|_{\Sigma_{a}^{j \pm 1} \cap \Omega_{a}^{j}}, \quad \forall j \in \mathbb{Z} / 4 \mathbb{Z} .
$$

The system of coupled equations (13) and (11) constitutes the halfspace matching formulation for the problem (4). To understand the nature of this system of equations and to analyze it, let us introduce the operators

$$
\forall \psi \in H^{1 / 2}\left(\Sigma_{a}^{j}\right), \quad D_{j \pm 1}^{j} \psi:=\left.P^{j}(\psi)\right|_{\Sigma_{a}^{j \pm 1} \cap \Omega_{a}^{j}} .
$$

By classical trace theorem, the operators $D_{j \pm 1}^{j}$ are continuous operators from $H^{1 / 2}\left(\Sigma_{a}^{j}\right)$ to $H^{1 / 2}\left(\Sigma_{a}^{j \pm 1} \cap \Omega_{a}^{j}\right)$. In the isotropic case, the expressions of the operators $D_{j \pm 1}^{j}$ derive directly from the expressions of two operators $D_{ \pm} \in$ $\mathcal{L}\left(H^{1 / 2}(\mathbb{R}), H^{1 / 2}(a,+\infty)\right)$ as follows

$$
\forall \psi \in H^{1 / 2}\left(\Sigma_{a}^{j}\right), \quad D_{j \pm 1}^{j} \psi^{j}\left(x_{j}, y_{j}=a\right)=D_{ \pm} \psi\left(x_{j}\right) \quad \text { for } x_{j}>a
$$

(identifying $H^{1 / 2}\left(\Sigma_{a}^{j}\right)$ to $H^{1 / 2}(\mathbb{R})$ ) where $D_{ \pm}$are defined by

$$
D_{ \pm} \psi(x)=\frac{1}{\sqrt{2 \pi}} \int_{\mathbb{R}} \widehat{\psi}(\xi) e^{\imath \sqrt{\omega_{\varepsilon}^{2}-\xi^{2}}(x-a)} e^{ \pm \imath \xi a} d \xi, \quad \text { for } x \geq a .
$$

Gathering (11), (13) and (14), we have shown that the set of traces

$$
\left(\varphi^{j}\right)_{j \in \llbracket 0,3 \rrbracket} \in \prod_{j=0}^{3} H^{1 / 2}\left(\Sigma_{a}^{j}\right)
$$


is solution of

$$
\forall j \in \mathbb{Z} / 4 \mathbb{Z}, \quad \mid \begin{array}{ll}
\varphi^{j}=D_{j}^{j-1} \varphi^{j-1} & \text { on } \Sigma_{a}^{j} \cap \Omega_{a}^{j-1}, \\
\varphi^{j}=D_{j}^{j+1} \varphi^{j+1} & \text { on } \Sigma_{a}^{j} \cap \Omega_{a}^{j+1}, \\
\left.\varphi^{j}\right|_{\Sigma_{a a}^{j}}=\left.g\right|_{\Sigma_{a a}^{j}} . &
\end{array}
$$

We have then derived a system of coupled integral equations satisfied by the traces $\varphi^{j}$ of the solution $p$ of (4). Moreover, there is an equivalence result between problem (4) and system (17). Indeed, from a solution $\left(\varphi^{j}\right)_{j \in \llbracket 0,3 \rrbracket}$ in $\prod H^{1 / 2}\left(\Sigma_{a}^{j}\right)$ of (17) and using the halfspace representations (9), we can construct a solution $p$ of (4). The only difficulty is to show that the halfspace representations match in their intersections - which are quarter planes. We can state then the following proposition whose proof is detailed in Section 3.

Proposition 2.1. Let $g \in H^{1 / 2}\left(\Sigma_{a a}\right)$. If $p \in H^{1}(\Omega)$ is solution of (4) then $\left(\varphi^{j}=\left.p\right|_{\Sigma_{a}^{j}}\right)_{j \in \llbracket 0,3 \rrbracket}$ is solution in $\prod H^{1 / 2}\left(\Sigma_{a}^{j}\right)$ of $(17)$.

Conversely, if $\left(\varphi^{j}\right)_{j \in \llbracket 0,3 \rrbracket}$ in $\prod H^{1 / 2}\left(\Sigma_{a}^{j}\right)$ is solution of $(17)$ then $p$ defined by

$$
\forall j \in \llbracket 0,3 \rrbracket,\left.\quad p\right|_{\Omega_{a}^{j}}=P^{j}\left(\varphi^{j}\right),
$$

where $P^{j}(\cdot)$ is solution of the halfspace problem (8) (see also the expression $(9))$, is a function defined "unequivocally", is in $H^{1}(\Omega)$ and is solution of (4).

This is the system (17) that we aim to discretize. If we want to use a FE method, a variational formulation has to be derived and using the functional framework $\prod H^{1 / 2}\left(\Sigma_{a}^{j}\right)$ could be intrincate. That is why we consider (17) when looking to the traces in $\prod L^{2}\left(\Sigma_{a}^{j}\right)$. The operators $D_{j \pm 1}^{j}$ which intervene in the formulation are well-defined and even continuous from $L^{2}\left(\Sigma_{a}^{j}\right)$ to $L^{2}\left(\Sigma_{a}^{j \pm 1} \cap \Omega_{a}^{j}\right)$ (see Section 3.2 for more details). Writing for all $j \in \llbracket 0,3 \rrbracket$, $\varphi^{j}=\varphi_{0}^{j}+\left.g\right|_{\Sigma_{a a}^{j}}$, we can easily show that Problem (17) in the $L^{2}$-framework is equivalent to

$$
\Phi_{0}=\left(\varphi_{0}^{j}\right)_{j \in \llbracket 0,3 \rrbracket} \in V_{0}=\left\{\Psi_{0}=\left(\psi_{0}^{j}\right) \in \prod_{j=0}^{3} L^{2}\left(\Sigma_{a}^{j}\right), \quad \forall j,\left.\psi_{0}^{j}\right|_{\Sigma_{a a}^{j}}=0\right\}
$$


is solution of

$$
\begin{gathered}
\mathbb{A} \Phi_{0}=\mathbb{B} g \\
\text { where } \quad \mathbb{A} \in \mathcal{L}\left(V_{0}\right), \mathbb{B} \in \mathcal{L}\left(L^{2}\left(\Sigma_{a a}\right), V_{0}\right) \text { and } \\
\forall j \in \mathbb{Z} / 4 \mathbb{Z}, \quad \mid \begin{array}{l}
\left(\mathbb{A} \Phi_{0}\right)_{j}=\varphi_{0}^{j}-D_{j}^{j-1} \varphi_{0}^{j-1}-D_{j}^{j+1} \varphi_{0}^{j+1} \\
(\mathbb{B} g)_{j}=\left.D_{j}^{j-1} g\right|_{\Sigma_{a a}^{j-1}}+\left.D_{j}^{j+1} g\right|_{\Sigma_{a a}^{j+1}}
\end{array}
\end{gathered}
$$

where for all $j$, we define the functions $D_{j}^{j \pm 1} \varphi_{0}^{j \pm 1},\left.D_{j}^{j \pm 1} g\right|_{\Sigma_{a a}^{j \pm 1}} \in L^{2}\left(\Sigma_{a}^{j} \cap\right.$ $\left.\Omega_{a}^{j \pm 1}\right)$ as functions of $L^{2}\left(\Sigma_{a}^{j}\right)$ by extending them by 0 . We are able to show stability property and well posedness of problem (20) in $V_{0}$.

Theorem 2.2 (Main result (Case 1)). (1) The operator $\mathbb{A} \in \mathcal{L}\left(V_{0}\right)$ is the sum of a coercive operator and a compact one. Thus, for Problem (20), Fredholm alternative holds.

(2) Problem (20) is well posed in $V_{0}$.

This Theorem will be shown in Section 3.

Remark 2.3. If $g \in H^{1 / 2}\left(\Sigma_{a a}\right)$, the last theorem gives that the problems (4) and (20) are equivalent. If the data $g$ is only in $L^{2}\left(\Sigma_{a a}\right)$, we cannot expect that the solution $p$ is in $H^{1}(\Omega)$. By using the transposition methods, in (21), the authors analyse how to understand the solution of such boundary value problem in bounded convex domain. They introduce a very weak formulation (the unknown is only in $L^{2}$ but the test functions is much more regular ). In recent papers (1; 2), an extension to non convex polygonal bounded domain which requires an involved analysis is proposed. For the particular case of Problem (20), we have introduced a simple formulation which is stable and whose unique solution is such that its trace on each line $\Sigma_{a}^{j}$ is $L^{2}$ (or equivalently its restriction to each halfspace $\Omega_{a}^{j}$ is $\left.H^{1 / 2}\right)$.

Let us point out that what is done here with $\Omega_{a}$ being a square can be extended naturally to the case of any convex polygon $\Omega_{a}$. The unknowns, which are involved in the corresponding system of equations, correspond to the traces of the solution on the boundary of the halfspaces supported by each edge of the polygon. See Appendix A for more details. 
2.2. Case 2 : coupling the halfspace matching method with the FE method in the isotropic case

We consider now problem (4), still in the isotropic case : $A_{0}=\mathrm{Id}$. Without loss of generality, for conciseness of the statement, we suppose $A=A_{0}$ and $\Omega=\mathbb{R}^{2}$ (it suffices to adapt the variational formulation and/or the functional framework to take into account the presence of perturbations, obstacles and the associated boundary conditions). Thus the problem simplifies to

$$
-\triangle p-\omega_{\varepsilon}^{2} \rho p=f \quad \text { in } \quad \mathbb{R}^{2}
$$

where $\operatorname{Supp}(\rho-1)$ and $\operatorname{Supp}(f)$ are included in $\Omega_{a}$.

Let us first introduce some new notations. For $b \geq a$, we denote (see Figure 2)

$$
\Omega_{b}=(-b, b)^{2}, \quad \Sigma_{b b}=\partial \Omega_{b} \quad \text { and } \quad \Sigma_{b b}^{j}=\left\{(x, y), x_{j}=b \text { and } y^{j} \in(-b, b)\right\}
$$

where the $\left(x^{j}, y^{j}\right)$ are defined in $(5)$.

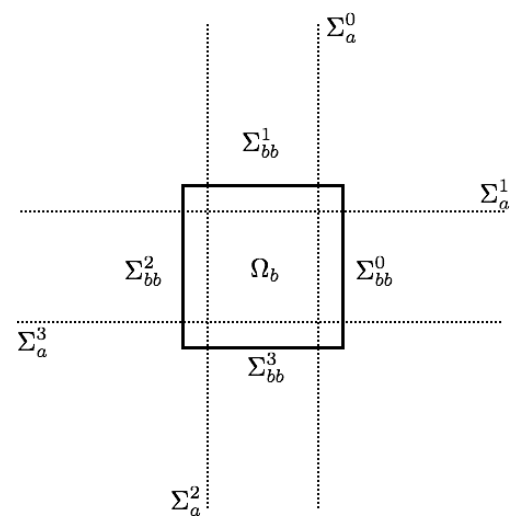

Figure 2: Second set of notations (22) (with the dashed lines are the $\Sigma_{a}^{j}$ 's defined in (6)).

In Figure 2, five domains appear: a bounded one $\Omega_{b}$ and the four halfspaces $\Omega_{a}^{j}$. If $b=a$, there is no overlap between the bounded domain and the halfspaces whereas if $b>a$, there exists an overlap. We will see that this overlap has an important role from a theoretical point of view.

Let us now derive the new formulation. Thanks to the previous section, it is easy to see that the traces $\varphi^{j}$ of $p$ on the lines $\Sigma_{a}^{j}$ have to satisfy (13). 
On the other hand, if $p_{b}$ denotes the restriction of $p$ to $\Omega_{b}$, then it is solution of

$$
\mid-\triangle p_{b}-\omega_{\varepsilon}^{2} \rho p_{b}=f \quad \text { in } \quad \Omega_{b} .
$$

For all $j$, in $\Omega_{b} \cap \Omega_{a}^{j}$, we have introduced two representations of the same function: $p^{b}$ solution of $(23)$ and $P^{j}\left(\varphi^{j}\right)$ solution of (8). We have then in particular that

$$
\left.\varphi^{j}\right|_{\Sigma_{a a}^{j}}=\left.p^{b}\right|_{\Sigma_{a a}^{j}}
$$

and

$$
\left.\nabla p \cdot n^{j}\right|_{\Sigma_{b b}^{j}}=\left.\nabla P^{j}\left(\varphi^{j}\right) \cdot n^{j}\right|_{\Sigma_{b b}^{j}},
$$

where $n^{j}=\left(x^{j}=1, y^{j}=0\right)$ is the normal to $\Sigma_{b b}^{j}$. Let us introduce the operators

$$
\forall j \in \llbracket 0,3 \rrbracket, \quad \forall \psi \in H^{1 / 2}\left(\Sigma_{a}^{j}\right), \quad \Lambda^{j} \psi=\left.\nabla P^{j}(\psi) \cdot n^{j}\right|_{\Sigma_{b b}^{j}}
$$

By classical trace theorem, the operators $\Lambda^{j}$ are continuous operators from $H^{1 / 2}\left(\Sigma_{a}^{j}\right)$ to $H^{-1 / 2}\left(\Sigma_{b b}^{j}\right)$ where $H^{-1 / 2}\left(\Sigma_{b b}^{j}\right)$ is defined as the dual of $\tilde{H}^{1 / 2}\left(\Sigma_{b b}^{j}\right)$ which contains the functions of $H^{1 / 2}\left(\Sigma_{b b}^{j}\right)$ which, when extending by 0 , are $H^{1 / 2}\left(\Sigma_{b b}\right)$. In the isotropic case, we have that for all $j \in \llbracket 0,3 \rrbracket, \Lambda^{j}$ can be expressed directly from the operator $\Lambda \in \mathcal{L}\left(H^{1 / 2}(\mathbb{R}), H^{-1 / 2}(-b, b)\right)$ by

$$
\forall \psi \in H^{1 / 2}\left(\Sigma_{a}^{j}\right), \quad \Lambda^{j} \psi\left(x^{j}=b, y^{j}\right)=\Lambda \psi\left(y^{j}\right) \quad \text { for } y^{j} \in(-b, b)
$$

(identifying $H^{1 / 2}\left(\Sigma_{a}^{j}\right)$ and $H^{1 / 2}(\mathbb{R})$ ) where $\Lambda$ is defined by

$$
\Lambda \psi(y)=\frac{1}{\sqrt{2 \pi}} \int_{\mathbb{R}} \imath \sqrt{\omega_{\varepsilon}^{2}-\xi^{2}} \widehat{\psi}(\xi) e^{\imath \sqrt{\omega_{\varepsilon}^{2}-\xi^{2}}(b-a)} e^{\imath \xi y} d \xi . \quad \text { for } y \in(-b, b) .
$$

Gathering (13), (14), (23), (24), (25) and (26) we have shown that

$$
p_{b} \in H^{1}\left(\Omega_{b}\right) \quad \text { and } \quad\left(\varphi^{j}\right)_{j \in \llbracket 0,3 \rrbracket} \in \prod_{j=0}^{3} H^{1 / 2}\left(\Sigma_{a}^{j}\right)
$$

is solution of

$$
\mid \begin{array}{ll}
-\triangle p_{b}-\omega_{\varepsilon}^{2} \rho p_{b}=f \text { in } \Omega_{b} & \\
\left.\nabla p \cdot n^{j}\right|_{\Sigma_{b b}^{j}}=\Lambda^{j} \varphi^{j}, \quad \forall j \in \llbracket 0,3 \rrbracket & \\
\left.\varphi^{j}\right|_{\Sigma_{a a}^{j}}=\left.p^{b}\right|_{\Sigma_{a a}^{j}}, \quad \forall j \in \llbracket 0,3 \rrbracket & \\
\varphi^{j}=D_{j}^{j-1} \varphi^{j-1} & \text { on } \Sigma_{a}^{j} \cap \Omega_{a}^{j-1}, \quad \forall j \in \mathbb{Z} / 4 \mathbb{Z} \\
\varphi^{j}=D_{j}^{j+1} \varphi^{j+1} & \text { on } \Sigma_{a}^{j} \cap \Omega_{a}^{j+1}, \quad \forall j \in \mathbb{Z} / 4 \mathbb{Z}
\end{array}
$$


There is here also an equivalence result between problem (4) in the isotropic case and the system (29). Indeed, from the trace unknowns, we can construct a solution $p_{\text {ext }}$ of the homogeneous isotropic Helmholtz equation outside $\Omega^{a}$, as in Case (1). Now the difficulty is to show that this function $p_{\text {ext }}$ coincides with $p_{b}$ in the overlapping zone $\Omega_{b} \backslash \Omega_{a}$, which will be shown in Section 4.1.

Proposition 2.4. If $p \in H^{1}(\Omega)$ is solution of $(21)$ then $\left(p_{b},\left(\varphi^{j}\right)_{j \in \llbracket 0,3 \rrbracket}\right)$ where $p_{b}=\left.p\right|_{\Omega_{b}}$ and for all $j, \varphi^{j}=\left.p\right|_{\Sigma_{a}^{j}}$ is solution in $H^{1}\left(\Omega_{b}\right) \times \prod H^{1 / 2}\left(\Sigma_{a}^{j}\right)$ of (29).

Conversely, if $\left(p_{b},\left(\varphi^{j}\right)_{j \in \llbracket 0,3 \rrbracket}\right)$ in $H^{1}\left(\Omega_{b}\right) \times \prod H^{1 / 2}\left(\Sigma_{a}^{j}\right)$ is solution of $(29)$ then $p$ defined by

$$
\left.p\right|_{\Omega_{b}}=p_{b}, \quad \text { and } \quad \forall j \in \llbracket 0,3 \rrbracket,\left.\quad p\right|_{\Omega_{a}^{j}}=P^{j}\left(\varphi^{j}\right),
$$

where $P^{j}(\cdot)$ is solution of the halfspace problem (8) (see also the expression (9)), is a function defined "unequivocally", is in $H^{1}(\Omega)$ and is solution of (21).

This is the system (29) that we aim to discretize and we have then to derive a variational formulation. However this is possible only for $b>a$, the problem, being as in (14), the cross points (see Section 4.2 for more details). Moreover, as for the case (1), we want to consider the trace unknowns in $L^{2}$ for numerical purposes. For $b>a$, the operators $\Lambda^{j}$ which intervene in the formulation are well-defined and continuous from $L^{2}\left(\Sigma_{a}^{j}\right)$ to $L^{2}\left(\Sigma_{b b}^{j}\right)$. Thus, for $b>a$, and by using, as for Case (1), for all $j, \varphi_{0}^{j}=\varphi^{j}-\left.p_{b}\right|_{\Sigma_{a a}^{j}}$, we introduce a variational formulation associated to (29):

Find $\left(p_{b}, \Phi=\left(\varphi_{0}^{j}\right)_{j \in \llbracket 0,3 \rrbracket}\right) \in H^{1}\left(\Omega_{b}\right) \times V_{0}$ such that $\forall\left(q_{b}, \Psi\right) \in H^{1}\left(\Omega_{b}\right) \times V_{0}$

$$
\begin{aligned}
\int_{\Omega_{b}} \nabla p_{b} & \nabla \overline{q_{b}}-\omega^{2} p_{b} \overline{q_{b}}+(\mathbb{A} \Phi, \Psi)_{V_{0}}-\left(\mathbb{B} \gamma p_{b}, \Psi\right)_{V_{0}} \\
& -\sum_{j=0}^{3}\left[\left(\Lambda^{j} \gamma^{j} p_{b}, q_{b}\right)_{\Sigma_{b b}^{j}}-\left(\Lambda^{j} \varphi_{0}^{j}, q_{b}\right)_{\Sigma_{b b}^{j}}\right]=\int_{\Omega_{b}} f \overline{q_{b}}
\end{aligned}
$$

where $V_{0}$ is defined in (19), the operators $\mathbb{A}$ and $\mathbb{B}$ are defined in (20), the operator $\gamma$ (resp. $\gamma^{j}$ ) is the trace operator from $H^{1}\left(\Omega_{b}\right)$ to $L^{2}\left(\Sigma_{a a}\right)$ (resp. from $H^{1}\left(\Omega_{b}\right)$ to $\left.L^{2}\left(\Sigma_{a a}^{j}\right)\right),(\cdot, \cdot)_{\Sigma_{b b}^{j}}$ is the Hermitian product in $L^{2}\left(\Sigma_{b b}^{j}\right),(\cdot, \cdot)_{V_{0}}$ is the Hermitian product in $\prod L^{2}\left(\Sigma_{a}^{j}\right)$ and where we define the functions of 
$L^{2}\left(\Sigma_{a a}^{j}\right), L^{2}\left(\Sigma_{a}^{j} \cap \Omega_{a}^{j \pm 1}\right)$ as functions of $L^{2}\left(\Sigma_{a}^{j}\right)$ when extending by 0.

Extending the proof of Theorem 2.2, we can show a stability result as well.

Theorem 2.5 (Main result (Case 2)). (1) The bilinear form associated to (31) in $\left(H^{1}\left(\Omega_{b}\right) \times V_{0}\right)^{2}$ is the sum of a coercive bilinear form and a compact one. Thus, for Problem (31), Fredholm alternative holds.

(2) Problem (31) is well posed and if $\left(p_{b}, \Phi_{0}=\left(\varphi_{0}^{j}\right)_{j \in \llbracket 0,3 \rrbracket}\right)$ in $H^{1}\left(\Omega_{b}\right) \times V_{0}$ is solution of (31) then $p$ defined as in (30) with for all $j, \varphi^{j}=\varphi_{0}^{j}+\left.p_{b}\right|_{\Sigma_{a a}^{j}}$ is a function defined "unequivocally", is in $H^{1}(\Omega)$ and is solution of $(21)$.

This Theorem will be shown in Section 4.2.

2.3. Case 3 : coupling the halfspace matching method and the FE method in the anisotropic case

Now, we consider Problem (1) in the general anisotropic case. Again without loss of generality, we suppose $\Omega=\mathbb{R}^{2}$. The method to derive the system of coupled equations is exactly the same than in Case (2), the only difference being the expression of the solution of the corresponding halfspace problems and then the expression of the integral operators defined in (14) and (26).

The solution $P^{j}(\psi) \in H^{1}\left(\Omega_{a}^{j}\right)$, for any $\psi \in H^{1 / 2}\left(\Sigma_{a}^{j}\right)$ of the halfspace problems

$$
\mid \begin{array}{ll}
-\nabla \cdot\left(A_{0} \nabla P^{j}\right)-\omega_{\varepsilon}^{2} P^{j}=0 & \text { in } \Omega_{a}^{j}, \\
P^{j}=\psi & \text { on } \Sigma_{a}^{j}
\end{array}
$$

is now given by

$$
P^{j}(\psi)\left(x^{j}, y^{j}\right)=\frac{1}{\sqrt{2 \pi}} \int_{\mathbb{R}} \hat{\psi}(\xi) e^{r^{j}(\xi)\left(x^{j}-a\right)} e^{i \xi y^{j}} d \xi, \quad \forall\left(x^{j}, y^{j}\right) \in[a,+\infty[\times \mathbb{R} .
$$

where the coefficients $r^{j}(\xi)$ are defined by :

$$
\begin{aligned}
& r^{0}(\xi)=r^{2}(\xi)=\frac{-i \xi c_{3}}{c_{1}}+i \sqrt{d_{1}(\xi)} \quad \text { with } \quad d_{1}(\xi)=\frac{\omega_{\varepsilon}^{2} c_{1}-d \xi^{2}}{\left(c_{1}\right)^{2}}, \\
& r^{1}(\xi)=r^{3}(\xi)=\frac{i \xi c_{3}}{c_{2}}+i \sqrt{d_{2}(\xi)} \quad \text { with } \quad d_{2}(\xi)=\frac{\omega_{\varepsilon}^{2} c_{2}-d \xi^{2}}{\left(c_{2}\right)^{2}} .
\end{aligned}
$$

where $d:=c_{1} c_{2}-\left(c_{3}\right)^{2}$. We deduce then the expressions of the operators $D_{j \pm 1}^{j}$ and $\Lambda^{j}$ for all $j \in \mathbb{Z} / 4 \mathbb{Z}$ defined respectively in (14) and (26). Contrary 
to the isotropic case, the operators are a priori different from each other. Using the same ideas than in Case (2), we can show that for $b>a$

$$
p_{b}=\left.p\right|_{\Omega_{b}} \in H^{1}\left(\Omega_{b}\right) \quad \text { and } \quad\left(\varphi^{j}=\left.p\right|_{\Sigma_{a}^{j}}\right)_{j \in \llbracket 0,3 \rrbracket} \in \prod_{j=0}^{3} L^{2}\left(\Sigma_{a}^{j}\right)
$$

is solution of (31) with for all $j, \varphi_{0}^{j}=\varphi^{j}-\left.p_{b}\right|_{\Sigma_{a a}^{j}}$ where, here, the operators are given by for all $\psi \in H^{1 / 2}\left(\Sigma_{a}^{j}\right)$

$$
D_{j \pm 1}^{j} \psi\left(x_{j}, y_{j}= \pm a\right)=\frac{1}{\sqrt{2 \pi}} \int_{\mathbb{R}} \widehat{\psi}(\xi) e^{r^{j}(\xi)\left(x_{j}-a\right)} e^{ \pm \imath \xi a} d \xi, \quad \text { for } x_{j} \geq a
$$

and

$$
\Lambda^{j} \psi\left(x_{j}=b, y_{j}\right)=\frac{1}{\sqrt{2 \pi}} \int_{\mathbb{R}} r^{j}(\xi) \widehat{\psi}(\xi) e^{r^{j}(\xi)(b-a)} e^{\imath \xi y} d \xi, \quad \text { for } y_{j} \in(-b, b)
$$

Proposition 2.4 extends easily to the anisotropic case. Indeed, as the reader will notice in the proof detailed in Section 4.1, we do not use the expression of the solution of the halfspace problems or the expression of the operators.

Let us now introduce the variational formulation

Find $\left(p_{b}, \Phi_{0}=\left(\varphi_{0}^{j}\right)_{j \in \llbracket 0,3 \rrbracket}\right) \in H^{1}\left(\Omega_{b}\right) \times V_{0}$ such that $\forall\left(q_{b}, \Psi_{0}\right) \in H^{1}\left(\Omega_{b}\right) \times V_{0}$

$$
\begin{aligned}
\int_{\Omega_{b}} A \nabla p_{b} & \cdot \nabla \overline{q_{b}}-\omega^{2} p_{b} \overline{q_{b}}+(\mathbb{A} \Phi, \Psi)_{V_{0}}-\left(\mathbb{B} \gamma p_{b}, \Psi\right)_{V_{0}} \\
& -\sum_{j=0}^{3}\left[\left(\Lambda^{j} \gamma^{j} p_{b}, q_{b}\right)_{\Sigma_{b b}^{j}}-\left(\Lambda^{j} \varphi_{0}^{j}, q_{b}\right)_{\Sigma_{b b}^{j}}\right]=\int_{\Omega_{b}} f \overline{q_{b}}
\end{aligned}
$$

where $V_{0}$ is defined in (19), the operators $\mathbb{A}$ and $\mathbb{B}$ are defined in (20), the operator $\gamma$ (resp. $\gamma^{j}$ ) is the trace operator from $H^{1}\left(\Omega_{b}\right)$ to $L^{2}\left(\Sigma_{a a}\right)$ (resp. from $H^{1}\left(\Omega_{b}\right)$ to $\left.L^{2}\left(\Sigma_{a a}^{j}\right)\right),(\cdot, \cdot)_{\Sigma_{b b}^{j}}$ is the Hermitian product in $L^{2}\left(\Sigma_{b b}^{j}\right),(\cdot, \cdot)_{V_{0}}$ is the Hermitian product in $\prod L^{2}\left(\Sigma_{a}^{j}\right)$ and where we define the functions of $L^{2}\left(\Sigma_{a a}^{j}\right), L^{2}\left(\Sigma_{a}^{j} \cap \Omega_{a}^{j \pm 1}\right)$ as functions of $L^{2}\left(\Sigma_{a}^{j}\right)$ when extending by 0 .

The main difference in the anisotropic case concerns the stability results of the variational formulation which are linked to the properties of the operators $D_{j \pm 1}^{j}$ and $\Lambda^{j}$. 
Theorem 2.6 (Main result (Case 3)). Let $b>a$ and $\theta$ defined by $\tan (\theta)=$ $\sqrt{c_{1} c_{2}-c_{3}^{2}} / c_{3}$ if $c_{3} \neq 0$ and $\theta=\pi / 2$ if $c_{3}=0$.

(i) If

$$
\max (\sin (\theta / 2), \cos (\theta / 2)) \max \left(\sqrt{c_{1}}, \sqrt{c_{2}}\right)<\left(c_{1} c_{2}-c_{3}^{2}\right)^{1 / 4},
$$

the bilinear form associated to (37) is the sum of a coercive bilinear form and a compact one in $\left(H^{1}\left(\Omega_{b}\right) \times V_{0}\right)^{2}$;

(ii) In the general case, Fredholm alternative holds for the problem (37) and it is well-posed.

This theorem will be established in Section 5. As you can notice, the first result was proved only for a certain class of moderate anisotropy. In particular if the $x^{0}$ and $y^{0}$ directions are chosen regarding the directions of anisotropy of $A_{0}$ then $c_{3}=0$ and the condition (38) reduces to

$$
\max \left(\frac{c_{1}}{c_{2}}, \frac{c_{2}}{c_{1}}\right)<4 \text {. }
$$

\subsection{Numerical results}

To end this section, let us briefly describe how to discretize this new formulation and show some numerical results to illustrate and validate the method.

For the approximation of the formulation (20) (resp. (31) and (37)), we use 1D Lagrange FE method for the trace unknowns (resp. 2D Lagrange FE method for the volumic unknown). More precisely, the finite dimensional space $V_{h, T} \subset V_{0}$ contains piecewise polynomial functions of $L^{2}(-T, T)^{4}$ where $T>0$. Let us emphasize that the numerical analysis at that point can be done directly thanks to the stability results (using the coercive+compact properties)

The difficulty in practice is to handle the integral operator terms. For instance, to compute

$$
\left(D^{+} \varphi, \psi\right)_{(a,+\infty)}=\frac{1}{\sqrt{2 \pi}} \int_{a}^{+\infty} \psi(x) \int_{-\infty}^{\infty} \widehat{\varphi}(\xi) e^{\imath \sqrt{\omega_{\epsilon}^{2}-\xi^{2}}(x-a)} e^{\imath \xi a} d \xi d x
$$

where $\varphi$ and $\psi$ are functions of $L^{2}(a,+\infty)$, 
- we reduce the inner integral in $\xi$ to the interval $\left[-T_{\xi}, T_{\xi}\right]$ where $T_{\xi}>0$,

- we use a quadrature formula to discretize the integral in $\xi$.

Doing so, the previous integral is approximated by

$$
\sum_{n=0}^{N_{\xi}} \frac{1}{\sqrt{2 \pi}} \widehat{\varphi}\left(\xi_{n}\right) e^{\imath \xi_{n} a} \int_{0}^{+\infty} e^{\imath \sqrt{\omega_{\epsilon}-\xi_{n}^{2}}(x-a)} \psi(x) d x
$$

where the $\left\{\xi_{n}\right\}_{n \in \llbracket 0, N_{\xi} \rrbracket}$ correspond to the quadrature nodes. Since $\varphi$ and $\psi$ are piecewise polynomial (and compactly supported), we can compute exactly $\widehat{\varphi}\left(\xi_{n}\right)$ and the integral term in $x$. We can treat similarly the integral terms related to the operators $D_{j \pm 1}^{j}$ and $\Lambda^{j}$. The complete numerical analysis of the method will be the topic of a forthcoming paper.

To validate the method, we have consider in Case (1) the particular data of the Hankel function on the boundary of the square:

$$
\left.p(x, y)\right|_{\Sigma_{a a}}=\left.\frac{1}{4 \imath} H\left(\omega_{\epsilon} \sqrt{x^{2}+y^{2}}\right)\right|_{\Sigma_{a a}} .
$$

In that situation, we can validate the results since we know the exact solution is given by $p(x, y)=\frac{1}{4} H\left(\omega_{\epsilon} \sqrt{x^{2}+y^{2}}\right)$. On Figure 3 (left), we have represented on $(-T, T)$ the real part of $\varphi^{0}$ and its approximation taking $\omega_{\epsilon}=10+0.001 \imath$ and using 1D P2 finite elements with $h=0.05, T=6$, $T_{\xi}=20$ and a Gauss quadrature formula of order 4 in a regular mesh of size 0.025 . We get a relative error in $L^{2}(-T, T)$ of $1.2 \%$. Let us emphasize that with this method, we can reconstruct the solution in the exterior domain. Indeed, we can compute a posteriori the solution in each halfspace $\Omega_{a}^{j}$ using the formulae (8) as represented on Figure 3 (right). Surprisingly, as we can see, even though the traces $\varphi^{j}$ are not close to zero at $y^{j}= \pm T$, the results are quite good.

Let us now show a numerical simulation in the anisotropic case. We have used the following parameters:

$$
\omega_{\epsilon}=10+0.001 \imath \text { and } A=\left[\begin{array}{cc}
1 & -0.8 \\
-0.8 & 1
\end{array}\right] \text {. }
$$

It is a case of strong anisotropy for which the condition (38) is not satisfied. We can see on Figure 4 that the method gives good numerical results. One simple way to validate these results is to see if the reconstructed solution in the halfspaces match in the quarters of plane. 

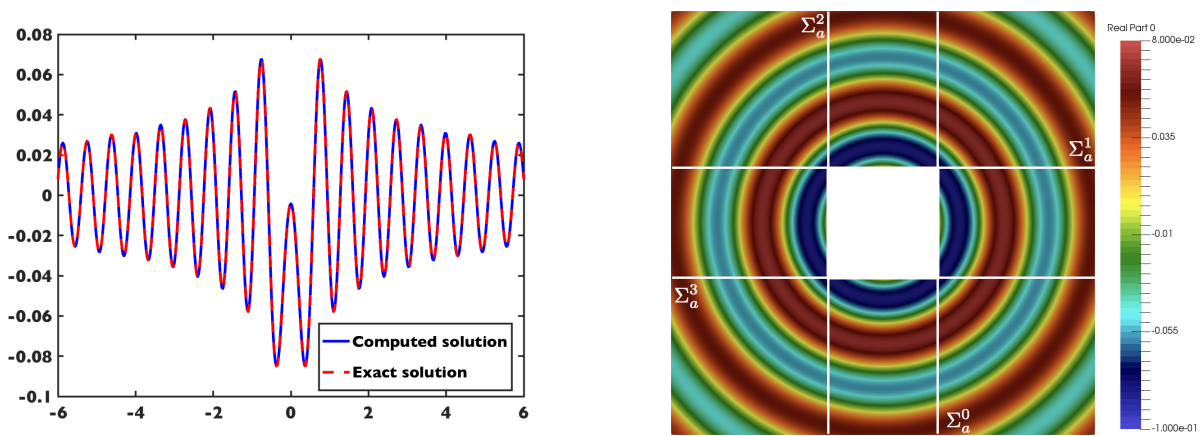

Figure 3: On the left: Real part of the computed trace $\varphi^{0}$ on $\Sigma_{a}^{0}$ (the black line) and real part of the exact solution (the dashed red line). On the right: Real part of the reconstructed solution in the exterior domain.

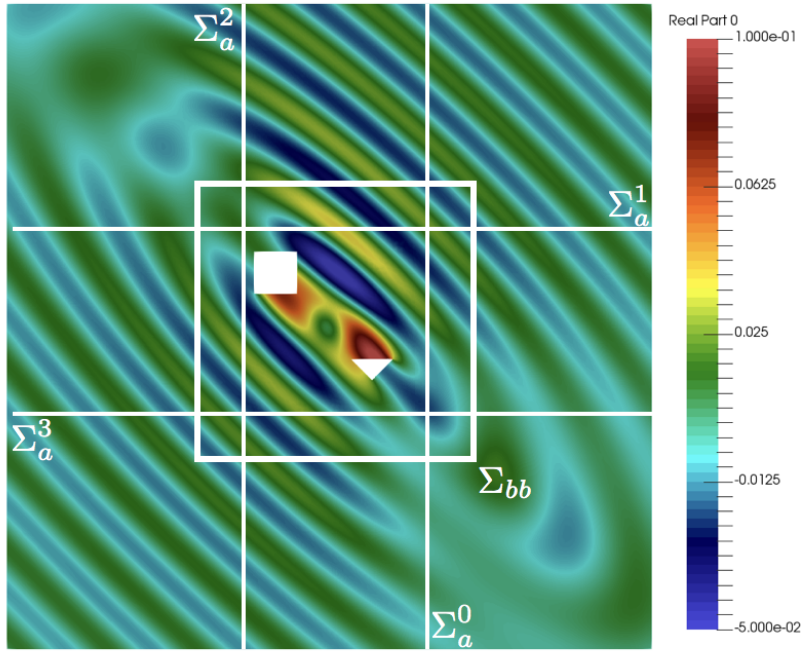

Figure 4: Real part of the computed solution in $\Omega_{b}$ and reconstruction of the solution in the halfspace $\Omega_{a}^{j}$. 


\section{Theoretical results related to the Case (1)}

In this section, we give the proof of Proposition 2.1 in Section 3.1 and the proof of Theorem 2.2 in Sections 3.2 and 3.3.

\subsection{The problem (4) and the formulation (17) are equivalent.}

Proposition 2.1 states that

1. the traces on $\Sigma_{a}^{j}$ of the solution of the original problem (4) are solution of the integral formulation (17): this is true by construction (see Section $2.1)$

2. from a solution of the integral formulation (17), we can reconstruct the solution of (4): this is proven in this section - for now the trace unknowns are in $H^{1 / 2}$.

Let us suppose that $\left(\varphi^{j}\right)_{j \in \llbracket 0,3 \rrbracket}$ in $\prod H^{1 / 2}\left(\Sigma_{a}^{j}\right)$ is solution of $(17)$. We want to reconstruct the solution $p$ in $H^{1}(\Omega)$ of (4).

For instance, from $\varphi^{0} \in H^{1 / 2}\left(\Sigma_{a}^{0}\right)$, we consider the corresponding halfspace solution $P^{0}\left(\varphi^{0}\right)$ in $H^{1}\left(\Omega_{a}^{0}\right)$ and from $\varphi^{1} \in H^{1 / 2}\left(\Sigma_{a}^{1}\right)$, we consider the corresponding halfspace solution $P^{1}\left(\varphi^{1}\right)$ in $H^{1}\left(\Omega_{a}^{1}\right)$. We claim that because $\varphi^{0}$ and $\varphi^{1}$ are solution of the first two equations of $(17), P^{0}\left(\varphi^{0}\right)$ and $P^{1}\left(\varphi^{1}\right)$ coincide in $\Omega_{a}^{0} \cap \Omega_{a}^{1}$. Indeed,

- by linearity the difference $V=P^{0}\left(\varphi^{0}\right)-P^{1}\left(\varphi^{1}\right)$ is a solution of the Helmholtz equation in the quarter plane

$$
-\Delta V-\omega_{\varepsilon}^{2} V=0 \quad \text { in } \quad \Omega_{a}^{0} \cap \Omega_{a}^{1} .
$$

- the first equation of (17) with $j=1$ is equivalent to saying that

$$
\left.\varphi^{1}\right|_{\Sigma_{a}^{1} \cap \Omega_{a}^{0}}=\left.P^{0}\left(\varphi^{0}\right)\right|_{\Sigma_{a}^{1} \cap \Omega_{a}^{0}}
$$

and by definition, we have $\varphi^{1}=\left.P^{1}\left(\varphi^{1}\right)\right|_{\Sigma_{a}^{1}}$. Then

$$
\left.V\right|_{\Sigma_{a}^{1} \cap \Omega_{a}^{0}}=\left.P^{0}\left(\varphi^{0}\right)\right|_{\Sigma_{a}^{1} \cap \Omega_{a}^{0}}-\left.P^{1}\left(\varphi^{1}\right)\right|_{\Sigma_{a}^{1} \cap \Omega_{a}^{0}}=0
$$

We deduce likewise -using the second equation with $j=0$ - that $\left.V\right|_{\Sigma_{a}^{0} \cap \Omega_{a}^{1}}=0$ and then

$$
\left.V\right|_{\partial\left(\Omega_{a}^{0} \cap \Omega_{a}^{1}\right)}=0
$$


So, by using (39) and (40), and by uniqueness of the quarter plane problem in $H_{0}^{1}$, we conclude that $V=0$ and that $P^{0}\left(\varphi^{0}\right)$ and $P^{1}\left(\varphi^{1}\right)$ coincide in $\Omega_{a}^{0} \cap \Omega_{a}^{1}$.

We can then define unequivocally a function $p$ by

$$
\left.p\right|_{\Omega_{a}^{0}}=P^{0}\left(\varphi^{0}\right) \text { and }\left.\quad p\right|_{\Omega_{a}^{1}}=P^{1}\left(\varphi^{1}\right) .
$$

Moreover, this function is solution in $H^{1}$ of the Helmholtz equation in $\Omega^{0} \cup \Omega^{1}$.

Using the other equations of (17) and the same kind of arguments, we can show that

$$
\forall j \in \mathbb{Z} / 4 \mathbb{Z}, \quad P^{j}\left(\varphi^{j}\right) \text { and } P^{j+1}\left(\varphi^{j+1}\right) \text { coincide on } \Omega_{a}^{j} \cap \Omega_{a}^{j+1}
$$

and then the function $p$ defined by

$$
\left.p\right|_{\Omega_{a}^{j}}=P^{j}\left(\varphi^{j}\right)
$$

is defined unequivocally and is solution in $H^{1}$ of the Helmholtz equation in $\Omega$. Finally,

$$
\forall j \in \llbracket 0,3 \rrbracket,\left.\quad p\right|_{\Sigma_{a a}^{j}}=\left.P^{j}\left(\varphi^{j}\right)\right|_{\Sigma_{a a}^{j}}=\left.g\right|_{\Sigma_{a a}^{j}}
$$

where the second inequality is deduced thanks to the third line of (17). This function $p$ is then solution of (4).

\subsection{Stability property of the integral formulation (20)}

We prove in this section, a part of Theorem 2.2 which states that the operator $\mathbb{A}$ defined in (20) is the sum of a coercive operator and a compact one. The well posedness of Problem (20) in $V_{0}$ will be proven in Section 3.3.

By (15) and (16), we can rewrite the operator $\mathbb{A}$ as

$$
\mathbb{A}=\mathbb{I}-\left[\begin{array}{cccc}
0 & J_{+}^{0} D_{-} & 0 & J_{-}^{0} D_{+} \\
J_{-}^{1} D_{+} & 0 & J_{+}^{1} D_{-} & 0 \\
0 & J_{-}^{2} D_{+} & 0 & J_{+}^{2} D_{-} \\
J_{+}^{3} D_{-} & 0 & J_{-}^{3} D_{+} & 0
\end{array}\right]
$$

where we have identified $L^{2}\left(\Sigma_{a}^{j}\right)$ and $L^{2}(\mathbb{R})$ and where for all $j \in \mathbb{Z} / 4 \mathbb{Z}, J_{ \pm}^{j}$ are unitary isomorphism from $L^{2}(a,+\infty)$ to $L^{2}\left(\Sigma_{a}^{j} \cap \Omega_{a}^{j \pm 1}\right)$ defined by

$$
\forall \psi \in L^{2}(a,+\infty), \quad J_{ \pm}^{j} \psi\left(x^{j}=a, y^{j}\right):=\psi\left( \pm y^{j}\right), \text { for } \pm y^{j} \geq a
$$


Let us note that $\forall j, J_{j}^{+}$is a canonical isomorphism and $J_{j}^{-}$makes the funtions run through $+\infty$ to $a$. Finally, we remind the reader that $D_{ \pm}$are operators from $L^{2}(\mathbb{R})$ to $L^{2}(a,+\infty)$ defined by

$$
\forall \varphi \in L^{2}(\mathbb{R}), \quad D_{ \pm} \varphi(x)=\frac{1}{\sqrt{2 \pi}} \int_{\mathbb{R}} \widehat{\varphi}(\xi) e^{\imath \sqrt{\omega_{\varepsilon}^{2}-\xi^{2}}(x-a)} e^{ \pm \imath \xi a} d \xi, \quad \text { for } x>a .
$$

It is quite obvious that the operators $D_{ \pm}$are continuous operators from $H^{1 / 2}(\mathbb{R})$ to $H^{1 / 2}(a,+\infty)$ (it suffices to use (14) where it is defined thanks to the trace operator). It is less obvious (but true) that the same property holds in the $L^{2}$ framework. To show this result, it is possible to use, as in (21), abstracts arguments: the operator being continuous from $H^{1 / 2}(\mathbb{R})$ to $H^{1 / 2}(a,+\infty)$, using a transposition argument, we can define it as a continuous operator from $H^{-1 / 2}(\mathbb{R})$ to $\left[H_{00}^{1 / 2}(a,+\infty)\right]^{\prime}$, and finally an interpolation argument gives that it is a continuous operator from $L^{2}(\mathbb{R})$ to $\left.L^{2}(a,+\infty)\right)$. A more constructive proof is provided in this section.

To show the stability property of Theorem 2.2, additional properties for the operators $D_{+}$and $D_{-}$are required. We first state these properties for $D_{+}$and give a detailed proof. Similar properties for $D_{-}$will be then given, without proofs, since similar arguments can be used.

Let us define for all interval $I \subset \mathbb{R}, R_{I}$ is a restriction operator of $L^{2}(\mathbb{R})$ defined by

$$
\forall \varphi \in L^{2}(\mathbb{R}), \quad R_{I} \varphi=\varphi \text { on } I \quad \text { and } \quad R_{I} \varphi=0 \text { on } \mathbb{R} \backslash I
$$

Proposition 3.1. The operator $D_{+}$is continuous from $L^{2}(\mathbb{R})$ to $L^{2}(a,+\infty)$. Moreover,

1. $D_{+} R_{(a,+\infty)}$ can be decomposed as

$$
D_{+} R_{(a,+\infty)}=L_{+} R_{(a,+\infty)}+K_{+}
$$

where $K_{+}$is a compact operator from $L^{2}(\mathbb{R})$ to $L^{2}(a,+\infty)$ and $L_{+}$is a continuous operator from $L^{2}(\mathbb{R})$ to $L^{2}(a,+\infty)$ which satisfies

$$
\forall \varphi \in L^{2}(\mathbb{R}), \quad\left\|L_{+} R_{(a,+\infty)} \varphi\right\|_{L^{2}(a,+\infty)} \leq \frac{1}{\sqrt{2}}\left\|R_{(a,+\infty)} \varphi\right\|_{L^{2}(\mathbb{R})} ;
$$

2. $D_{+} R_{(-\infty,-a)}$ is a compact operator from $L^{2}(\mathbb{R})$ to $L^{2}(a,+\infty)$. 
The main difficulty in the analysis is that $D_{+}$is not a compact operator. Going back to the halfspace problem, $D_{+}$is a trace operator on a half-line touching the boundary of the halfspace. The lack of compactness comes from this intersection point. To analyse the non-compact part and inspired by singularity theory (20), we introduce the static equivalent of $D^{+}$for $\omega_{\varepsilon}=0$, denoted by $L$ below. This strategy is often used in Integral Equation analysis, see for instance (5).

By decomposing $D_{+}$as

$$
D_{+}=R_{(a, b)} D_{+}+R_{(b,+\infty)} D_{+}
$$

the proof of Proposition 3.1 follows from the following properties

- the operator $R_{(b,+\infty)} D_{+}: L^{2}(\mathbb{R}) \rightarrow L^{2}(a,+\infty)$ is a compact operator;

- the operator $R_{(a, b)} D_{+}=R_{(a, b)} L+K_{1}$ where $L$ is a continuous operator from $L^{2}(\mathbb{R})$ to $L^{2}(a,+\infty)$ and $K_{1}$ is a compact operator from $L^{2}(\mathbb{R})$ to $L^{2}(a,+\infty)$;

- $\forall \varphi \in L^{2}(\mathbb{R}), \quad\left\|L R_{(a,+\infty)} \varphi\right\|_{L^{2}(a,+\infty)} \leq \frac{1}{\sqrt{2}}\left\|R_{(a,+\infty)} \varphi\right\|_{L^{2}(\mathbb{R})} ;$

- $L R_{(-\infty,-a)}$ is a compact operator from $L^{2}(\mathbb{R})$ to $L^{2}(a,+\infty)$

Lemma 3.2. The operator $R_{(b,+\infty)} D_{+}: L^{2}(\mathbb{R}) \rightarrow L^{2}(a,+\infty)$ is a compact operator.

Proof: Let us notice that $D_{+}$is a kernel operator

$$
\forall \psi \in L^{2}\left(\Sigma_{a}^{0}\right), \quad D_{+} \psi(x)=\frac{1}{\sqrt{2 \pi}} \int_{\mathbb{R}} \widehat{\psi}(\xi) k_{+}(x, \xi) d \xi, \quad \text { for } x \geq a,
$$

with the kernel

$$
k_{+}(x, \xi)=e^{\imath \sqrt{\omega_{\varepsilon}^{2}-\xi^{2}}(x-a)} e^{\imath \xi a} .
$$

Since $b>a$, this kernel is square-integrable

$$
\int_{\mathbb{R}} \int_{b}^{+\infty}\left|k_{+}(x, \xi)\right|^{2} d x d \xi=\int_{\mathbb{R}} \frac{e^{-2 \operatorname{Im}\left(\sqrt{\omega_{\varepsilon}^{2}-\xi^{2}}\right)(b-a)}}{2 \operatorname{Im}\left(\sqrt{\omega_{\varepsilon}^{2}-\xi^{2}}\right)}<+\infty
$$

Classical results on Hilbert-Schmidt operators gives that $R_{(b,+\infty)} D_{+}$is compact. 
Lemma 3.3. The operator $R_{(a, b)} D_{+}: L^{2}(\mathbb{R}) \rightarrow L^{2}(a,+\infty)$ can be written

$$
R_{(a, b)} D_{+}=R_{(a, b)} L+K_{1}
$$

where $L$ is a continuous operator from $L^{2}(\mathbb{R})$ to $L^{2}(a,+\infty)$ and $K_{1}$ is a compact operator from $L^{2}(\mathbb{R})$ to $L^{2}(a,+\infty)$. Moreover

1. L is bounded by 1 ;

2. $\forall \varphi \in L^{2}(\mathbb{R}), \quad\left\|L R_{(a,+\infty)} \varphi\right\|_{L^{2}(a,+\infty)} \leq \frac{1}{\sqrt{2}}\left\|R_{(a,+\infty)} \varphi\right\|_{L^{2}(\mathbb{R})} ;$

3. $R_{(a, b)} L R_{(-\infty,-a)}$ is a compact operator from $L^{2}(\mathbb{R})$ to $L^{2}(a,+\infty)$

Proof: Let $\varphi$ be in $L^{2}(\mathbb{R})$ and let us introduce $v(\varphi)$ a solution in the distributional sense of

$$
\begin{cases}-\triangle v=0, & \text { in } \Omega_{a}^{0} \\ v(\varphi)=\varphi, & \text { on } x=a\end{cases}
$$

We use in what follows the Mellin Transform (see for instance (20) for more details). Denoting $(r, \theta)$ the polar coordinates defined by (see Figure 5 )

$$
\mid \begin{aligned}
& r=\sqrt{(x-a)^{2}+(y-a)^{2}} \in(0,+\infty) \\
& \theta=\frac{\pi}{2}+\arctan \left(\frac{y-a}{x-a}\right) \in(0, \pi)
\end{aligned}
$$

we introduce the function $w$ defined for a.e. $(t, \theta) \in \mathcal{B} \equiv \mathbb{R} \times(0, \pi)$ by $w(t, \theta)=v(x, y)$ where $t=\log (r)$ and $(r, \theta)$ defined in (48). The function $w$ is a solution in the distributional sense of

$$
\left\{\begin{array}{l}
-\triangle w=0 \quad \text { in } \mathcal{B} \\
w(t, 0)=w_{0}(t):=\varphi\left(-e^{t}+a\right) \\
w(t, \pi)=w_{\pi}(t):=\varphi\left(e^{t}+a\right)
\end{array}\right.
$$




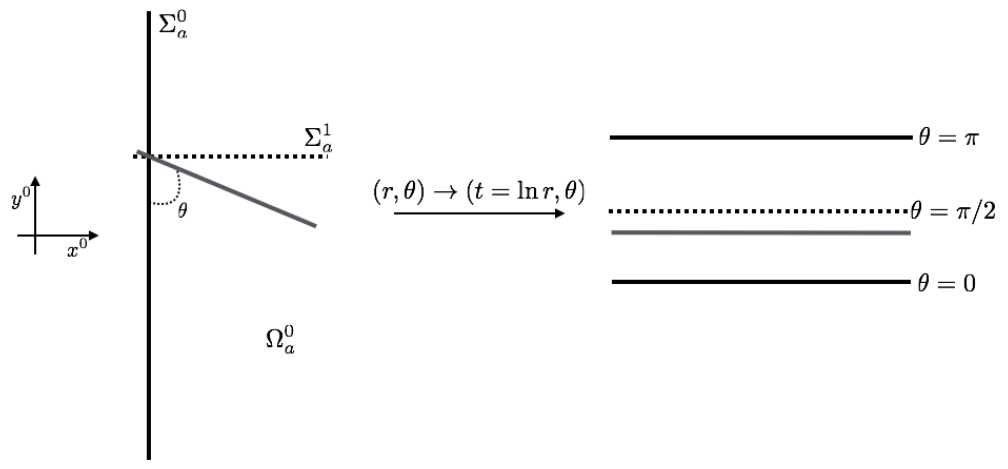

Figure 5: Notations and change of variables

Since $\varphi$ is in $L^{2}(\mathbb{R})$, we have $t \mapsto e^{t / 2} w_{0}(t)$ and $t \mapsto e^{t / 2} w_{\pi}(t)$ in $L^{2}(\mathbb{R})$ and

$$
\left\|e^{t / 2} w_{0}(t)\right\|_{L^{2}(\mathbb{R})}=\|\varphi\|_{L^{2}(-\infty, a)} \quad \text { and }\left\|e^{t / 2} w_{\pi}(t)\right\|_{L^{2}(\mathbb{R})}=\|\varphi\|_{L^{2}(a,+\infty)}
$$

To compute $w$, we can use the Fourier-Laplace transform defined by

$$
\hat{u}(\lambda) \equiv\left[\mathcal{M}_{t \rightarrow \lambda} u\right](\lambda):=\int_{\mathbb{R}} e^{-\lambda t} u(t) d t .
$$

It is an isomorphism between $\left\{u, e^{\beta t} u \in L^{2}(\mathbb{R})\right\}$ and $L^{2}\left(\ell_{-\beta}\right)$ where $\ell_{-\beta}=$ $\{\lambda=-\beta+\imath s, s \in \mathbb{R}\}$, for all $\beta \in \mathbb{R}$ and we have the Plancherel formula

$$
\int_{\mathbb{R}} e^{2 \beta t}|u(t)|^{2} d t=\frac{1}{2 \imath \pi} \int_{\ell_{-\beta}}|\hat{u}(\lambda)|^{2} d \lambda:=\|\hat{u}\|_{L^{2}\left(\ell_{-\beta}\right)}^{2} .
$$

We have in particular thanks to (50)

$$
\mid \begin{aligned}
& \hat{w}_{0} \in L^{2}\left(\ell_{-1 / 2}\right), \quad\left\|\hat{w}_{0}\right\|_{L^{2}\left(\ell_{-1 / 2}\right)}=\|\varphi\|_{L^{2}(-\infty, a)} \\
& \hat{w}_{\pi} \in L^{2}\left(\ell_{-1 / 2}\right), \quad\left\|\hat{w}_{\pi}\right\|_{L^{2}\left(\ell_{-1 / 2}\right)}=\|\varphi\|_{L^{2}(a,+\infty)}
\end{aligned}
$$

Applying this transformation to (49) and using the classical derivation rule of the Fourier transform, we show that $\theta \rightarrow \hat{w}(\lambda, \theta)$ is solution of an ODE parametrized by $\lambda$. Solving this ODE, we obtain for $\lambda \notin \mathbb{Z}$

$$
\forall \theta \in(0, \pi), \quad \hat{w}(\lambda, \theta)=A(\lambda, \pi-\theta) \hat{w}_{0}(\lambda)+A(\lambda, \theta) \hat{w}_{\pi}(\lambda)
$$

where

$$
A(\lambda, \theta)=\frac{\sin (\lambda \theta)}{\sin (\lambda \pi)}
$$


For all $\theta \in(0, \pi), s \mapsto A(-1 / 2+\imath s, \theta)$ is in $L^{\infty}(\mathbb{R})$, its supremum is attained for $s=0$ and it is equal to $\sin (\theta / 2)$. In particular for $\theta=\pi / 2$, we have

$$
\sup _{-1 / 2+\imath \mathbb{R}}|A(\lambda, \pi / 2)|=\frac{1}{\sqrt{2}}
$$

and then

$$
\|\hat{w}(\lambda, \pi / 2)\|_{L^{2}\left(\ell_{-\beta}\right)} \leq \frac{1}{\sqrt{2}}\left[\left\|\hat{w}_{0}\right\|_{L^{2}\left(\ell_{-1 / 2}\right)}+\left\|\hat{w}_{\pi}\right\|_{L^{2}\left(\ell_{-1 / 2}\right)}\right] .
$$

We deduce using successively (53), (50), (52) and the change of variables $r=e^{t}$ that

$$
\left.v(\varphi)\right|_{y=a} \in L^{2}(a,+\infty) \quad \text { and } \quad\left\|\left.v(\varphi)\right|_{y=a}\right\|_{L^{2}(a,+\infty)} \leq\|\varphi\|_{L^{2}(\mathbb{R})}
$$

The operator $L$ defined by

$$
\forall \varphi \in L^{2}(\mathbb{R}), \quad L \varphi=\left.v(\varphi)\right|_{y=a}
$$

is then a continuous operator from $L^{2}(\mathbb{R})$ to $L^{2}(a,+\infty)$ and its norm is bounded by 1 (this bound is optimal but we do not prove it here since it is not useful for the following).

Moreover, using that

$$
\sup _{\theta \in(0, \pi)} \sup _{-1 / 2+\imath \mathbb{R}}|A(\lambda, \theta)|=1
$$

we show with the same tools that

$$
\begin{aligned}
\int_{0}^{\pi} \int_{\mathbb{R}^{+}}|v(r, \theta)|^{2} d r d \theta=\int_{0}^{\pi} & \int_{\mathbb{R}}|w(t, \theta)|^{2} e^{t} d t d \theta \\
& =\int_{0}^{\pi} \int_{\ell_{-1 / 2}}|\hat{w}(\lambda, \theta)|^{2} d \lambda d \theta \leq 2\|\varphi\|_{L^{2}(\mathbb{R})}^{2} .
\end{aligned}
$$

This means that $r^{-1 / 2} v \in L^{2}\left(\Omega_{a}\right)$. Thus $v$ is not in $L^{2}\left(\Omega_{a}\right)$ but it is $L_{\text {loc }}^{2}\left(\Omega_{a}\right)$ and for any compact set $K \subset \Omega_{a}$, we have

$$
\exists C>0, \quad\|v\|_{L^{2}(K)} \leq C\|\varphi\|_{L^{2}(\mathbb{R})} .
$$


By noticing that $L \varphi$ is also given by

$$
L \varphi(x)=\frac{1}{\sqrt{2 \pi}} \int_{\mathbb{R}} \hat{\varphi}(\xi) e^{-|\xi|(x-a)} e^{\imath \xi a}
$$

we can show using the same arguments than in the proof of Lemma 3.2 that $R_{(a, b)}\left(D_{+} \varphi-L \varphi\right)$ is a compact operator.

The property on $L R_{(a,+\infty)}$ can be deduced from the previous computation by taking $\left.\varphi\right|_{(-\infty, a)}=0$ or equivalently $\hat{w}_{0}=0$. From the estimation (56), we can conclude that

$$
\forall \varphi \in L^{2}(\mathbb{R}), \quad\left\|L R_{(a,+\infty)} \varphi\right\|_{L^{2}(a,+\infty)} \leq \frac{1}{\sqrt{2}}\left\|R_{(a,+\infty)} \varphi\right\|_{L^{2}(\mathbb{R})} .
$$

Finally, let us consider the previous computations with $\varphi=0$ on $(-a,+\infty)$. The function $w$ is solution of (49) with $w_{\pi}=0$ and $w_{0}(t)=\varphi\left(-e^{t}+a\right)$. Since $\varphi(y)=0$ for $y>-a$, we have $e^{-\gamma t} w_{0}$ is in $L^{2}(\mathbb{R})$ for all $\gamma>0$. By using the Fourier Laplace Transform defined in (51), $\hat{w}_{0}$ is in $L^{2}\left(\ell_{\gamma}\right)$ for all $\gamma>0$. Moreover, again as in the proof of Lemma 3.3, for $\lambda \notin \mathbb{Z}$

$$
\hat{w}(\lambda, \theta)=A(\lambda, \theta) \hat{w}_{0}(\lambda)
$$

We can show that

$$
\forall \gamma>0, \gamma \notin \mathbb{N} \quad \sup _{\ell_{\gamma}} \lambda A(\lambda, \theta)<+\infty
$$

which enables us to deduce that

$$
\forall \gamma>0, \gamma \notin \mathbb{N} \quad \lambda \hat{w}(\lambda, \theta) \in L^{2}\left(\ell_{\gamma}\right)
$$

In particular for $\theta=\pi / 2$ and by using the properties of the Fourier-Laplace Transform, we have then

$$
\forall \gamma>0, \gamma \notin \mathbb{N} \quad e^{-\gamma t} \partial_{t} w(t, \pi / 2) \in L^{2}(\mathbb{R})
$$

which gives

$$
\forall \gamma>0, \quad r^{-(\gamma+1 / 2)}\left(r \partial_{r}\right) v(r, \pi / 2) \in L^{2}(a,+\infty)
$$

If we choose $\gamma=1 / 2$, we obtain that $L R_{(-\infty,-a)} \varphi=v(r, \pi / 2) \in H^{1}(a,+\infty)$. By compact embedding of $H^{1}(a, b)$ in $L^{2}(a, b)$, we conclude that $R_{(a, b)} L R_{(-\infty,-a)}$ 
is a compact operator from $L^{2}(\mathbb{R})$ to $L^{2}(a,+\infty)$.

Using the same arguments, we can show the following properties for $D_{-}$.

Proposition 3.4. The operator $D_{-}$is continuous from $L^{2}(\mathbb{R})$ to $L^{2}(a,+\infty)$. Moreover,

1. $D_{-} R_{(-\infty,-a)}$ can be decomposed as

$$
D_{-} R_{(-\infty,-a)}=L_{-} R_{(-\infty,-a)}+K_{-}
$$

$K_{-}$is a compact operator from $L^{2}(\mathbb{R})$ to $L^{2}(a,+\infty)$ where $L_{-}$is a continuous operator from $L^{2}(\mathbb{R})$ to $L^{2}(a,+\infty)$ which satisfies

$$
\forall \varphi \in L^{2}(\mathbb{R}), \quad\left\|L_{-} R_{(-\infty,-a)} \varphi\right\|_{L^{2}(a,+\infty)} \leq \frac{1}{\sqrt{2}}\left\|R_{(-\infty,-a)} \varphi\right\|_{L^{2}(\mathbb{R})} ;
$$

2. $D_{-} R_{(a,+\infty)}$ is a compact operator from $L^{2}(\mathbb{R})$ to $L^{2}(a,+\infty)$.

Using the previous results, we can now prove that the operator $\mathbb{A} \in \mathcal{L}\left(V_{0}\right)$, described in (41), is the sum of a coercive operator and a compact one. Indeed, using Propositions 3.1 and 3.4, the operator $\mathbb{A}$ can be decomposed as

$$
\mathbb{I}-\mathbb{L}-\mathbb{K}
$$

where

$$
\mathbb{L}=\left[\begin{array}{cccc}
0 & J_{+}^{0} L_{-} R_{(-\infty,-a)} & 0 & J_{-}^{0} L_{+} R_{(a,+\infty)} \\
J_{-}^{1} L_{+} R_{(a,+\infty)} & 0 & J_{+}^{1} L_{-} R_{(-\infty,-a)} & 0 \\
0 & J_{-}^{2} L_{+} R_{(a,+\infty)} & 0 & J_{+}^{2} L_{-} R_{(-\infty,-a)} \\
J_{+}^{3} L_{-} R_{(-\infty,-a)} & 0 & J_{-}^{3} L_{+} R_{(a,+\infty)} & 0
\end{array}\right]
$$

and

$$
\mathbb{K}=\left[\begin{array}{cccc}
0 & J_{+}^{0} \tilde{K}_{-} & 0 & J_{-}^{0} \tilde{K}_{+} \\
J_{-}^{1} \tilde{K}_{+} & 0 & J_{+}^{1} \tilde{K}_{-} & 0 \\
0 & J_{-}^{2} \tilde{K}_{+} & 0 & J_{+}^{2} \tilde{K}_{-} \\
J_{+}^{3} \tilde{K}_{-} & 0 & J_{-}^{3} \tilde{K}_{+} & 0
\end{array}\right]
$$

where the $J_{ \pm}^{j}$ are unitary isomorphism defined in $(42), \tilde{K}_{+}=D_{+} R_{(-\infty,-a)}+$ $K_{+} R_{(a,+\infty)}$ and $\tilde{K}_{-}=D_{-} R_{(a,+\infty)}+K_{-} R_{(-\infty,-a)}$ are compact operators of 
$L^{2}(\mathbb{R})$, by Proposition 3.1 and 3.4. $\mathbb{K}$ is then a compact operator of $\prod L^{2}\left(\Sigma_{a}^{j}\right)$. Moreover, it is easy to see that the norm of $\mathbb{L}$ as an operator of $V_{0}$ is bounded by $1 / \sqrt{2}$ by noting that for all $j \in \mathbb{Z} / 3 \mathbb{Z}$ and for all $\varphi^{j+1} \in L^{2}\left(\Sigma_{a}^{j+1}\right)$ and $\varphi^{j-1} \in L^{2}\left(\Sigma_{a}^{j-1}\right)$,

$$
\left\|J_{+}^{j} L_{-} R_{(-\infty,-a)} \varphi^{j+1}+J_{-}^{j} L_{+} R_{(a,+\infty)} \varphi^{j-1}\right\|^{2} \leq \frac{1}{2}\left(\left\|R_{(a,+\infty)} \varphi^{j-1}\right\|^{2}+\left\|R_{(-\infty,-a)} \varphi^{j+1}\right\|^{2}\right)
$$

so that

$$
\|\mathbb{L} \Phi\|^{2} \leq \frac{1}{2} \sum_{j=0}^{3}\left(\left\|R_{(a,+\infty)} \varphi^{j}\right\|^{2}+\left\|R_{(-\infty,-a)} \varphi^{j}\right\|^{2}\right)
$$

Consequently, $\mathbb{I}-\mathbb{L}$ is coercive in $V_{0}$.

\subsection{Well posedness of the integral formulation (20)}

As the operator $\mathbb{A}$ involved in (20) is a Fredholm operator of index 0 , it suffices to show its injectivity to deduce its surjectivity.

Similarly to Section 3.1, let us suppose that $\left(\varphi^{j}\right)_{j \in \llbracket 0,3 \rrbracket}$ is solution of Problem (20) with $g=0$ but here it is only in $\prod L^{2}\left(\Sigma_{a}^{j}\right)$. By reconstructing a solution of (4) with $g=0$, we want to show that $\left(\varphi^{j}\right)_{j \in \llbracket 0,3 \rrbracket}=0$. The difficulty here is that the corresponding halfspace solutions are not in $H^{1}$ since the $\varphi_{j}$ 's are not in $H^{1 / 2}$ but in $L^{2}$.

From $\varphi^{0} \in L^{2}\left(\Sigma_{a}^{0}\right)$ (resp. $\varphi^{1} \in L^{2}\left(\Sigma_{a}^{1}\right)$ ), we consider the halfspace function $P^{0}\left(\varphi^{0}\right)$ (resp. $P^{1}\left(\varphi^{1}\right)$ ) defined in (9) which is only in $H^{1 / 2}\left(\Omega_{a}^{0}\right)$ (resp. $\left.H^{1 / 2}\left(\Omega_{a}^{1}\right)\right)$. It is the solution of the corresponding halfspace problem in $\Omega_{a}^{0}$ (resp. $\Omega_{a}^{1}$ ) in a very weak sense (see (21)). Because of this non variational framework, it is less obvious than in Section 3.1 to prove that because $\varphi^{0}$ and $\varphi^{1}$ are solution of the first two equations of $(17), P^{0}\left(\varphi^{0}\right)$ and $P^{1}\left(\varphi^{1}\right)$ coincide in $\Omega_{a}^{0} \cap \Omega_{a}^{1}$. Nevertheless, we can show that the difference $V=P^{0}\left(\varphi^{0}\right)-P^{1}\left(\varphi^{1}\right) \in L^{2}\left(Q_{a}^{01}\right)$ with $Q_{a}^{01}=\Omega_{a}^{0} \cap \Omega_{a}^{1}$ is solution in the distributional sense of the Helmholtz equation

$$
-\Delta V-\omega_{\varepsilon}^{2} V=0 \text { in } Q_{a}^{01} .
$$

Moreover, the "trace" of $\mathrm{V}$ vanishes on the boundary of the quarter-plane in the sense that

$$
\forall u \in H^{2}\left(Q_{a}^{01}\right) \cap H_{0}^{1}\left(Q_{a}^{01}\right), \quad \int_{Q_{a}^{01}} \triangle V u=\int_{Q_{a}^{01}} V \triangle u .
$$


We deduce that

$$
0=\int_{Q_{a}^{01}}\left(\triangle V+\omega_{\varepsilon}^{2} V\right) u=\int_{Q_{a}^{01}} V\left(\triangle u+\omega_{\varepsilon}^{2} u\right) .
$$

Using classical results of regularity of solutions in convex polygonal domains (see for instance (17)), we know that the operator

$$
\begin{aligned}
A_{a}^{01}: H^{2}\left(Q_{a}^{01}\right) \cap H_{0}^{1}\left(Q_{a}^{01}\right) & \rightarrow L^{2}\left(Q_{a}^{01}\right) \\
u & \mapsto \triangle u+\omega_{\varepsilon}^{2} u
\end{aligned}
$$

is surjective. We conclude that $V=0$ and then $P^{0}\left(\varphi^{0}\right)$ and $P^{1}\left(\varphi^{1}\right)$ coincide in the quarter plane $Q_{a}^{01}$.

Using the other equations of (17) and the same kind of arguments, we can show that the function $p \in L^{2}(\Omega)$ defined by

$$
\left.p\right|_{\Omega_{a}^{j}}=P^{j}\left(\varphi^{j}\right)
$$

is defined unequivocally. Let us prove now that $p=0$. By definition, it is solution in the distributional sense of the Helmholtz equation in $\Omega$ and its trace on each line $\Sigma_{a}^{j}$ are in $L^{2}$. Moreover, we can show that its "trace" on $\partial \Omega_{a}$ vanishes in the sense that

$$
\forall u \in H^{2}(\Omega) \cap H_{0}^{1}(\Omega), \quad \int_{\Omega} \triangle p u=\int_{\Omega} p \triangle u .
$$

Indeed, we have thanks to (17, Theorem 1.5.3) that (62) holds for any $u \in H^{2}(\Omega) \cap H_{0}^{1}(\Omega)$ which vanishes near the corners and the subset of $H^{2}(\Omega) \cap H_{0}^{1}(\Omega)$ of functions which vanishes near the corners is dense in $H^{2}(\Omega) \cap H_{0}^{1}(\Omega)$ by extending the proof of Lemma 2.1.2 of (17).

We deduce that the function p satisfies for all $u \in H^{2}(\Omega) \cap H_{0}^{1}(\Omega)$,

$$
0=\int_{\Omega}\left(\triangle p+\omega_{\varepsilon}^{2} p\right) u=\int_{\Omega} p\left(\triangle u+\omega_{\varepsilon}^{2} u\right) .
$$

The function $p$ is then orthogonal to the range of the operator

$$
\begin{aligned}
A: H^{2}(\Omega) \cap H_{0}^{1}(\Omega) & \rightarrow L^{2}(\Omega) \\
u & \mapsto \triangle u+\omega_{\varepsilon}^{2} u .
\end{aligned}
$$


Using (17, Section 2.3), $p$ can then be decomposed as

$$
p_{R}+\sum_{j=0}^{3} \alpha_{j} r_{j}^{-2 / 3} \sin \left(2 \theta_{j} / 3\right)
$$

where $\left(r_{j}, \theta_{j}\right)$ are the polar coordinates whose origin is the corner $\left(x_{j}=\right.$ $\left.a, y_{j}=a\right)$ and such that $\theta_{j}$ is the angle from $\Sigma_{a a}^{j}$. The restriction of $p$ to each halfspace $\Omega_{a}^{j}$ being in $H^{1 / 2}\left(\Omega_{a}^{j}\right)$ (or equivalently the "trace" of $p$ to each line $\Sigma_{a}^{j}$ being in $\left.L^{2}\left(\Omega_{a}^{j}\right)\right)$, we conclude that each $\alpha_{j}=0$ and then $p$ is in $H^{1}(\Omega)$. As a solution of the Helmholtz equation in $\Omega$ whose trace vanishes on $\partial \Omega, p$ is equal to 0 and its traces on each $\Sigma_{a}^{j}$ as well : $\varphi^{j}=0$.

\section{Theoretical results associated to Case (2)}

In this section, we give the proof of Proposition 2.4 in Section 4.1 and Theorem 2.5 in Sections 4.2.

\subsection{Problem (1) in the isotropic case and Problem (29) are equivalent}

Proposition 2.4 states that

1. the restriction in $\Omega_{b}$ and the traces on $\Sigma_{a}^{j}$ of the solution of the original problem (4) are solution of Problem (29): this is true by construction;

2. from a solution of Problem (29), we can reconstruct the solution of (1): this is proven in Section 4.1 - for now the trace unknowns are in $H^{1 / 2}$.

Let us suppose that $\left(p_{b},\left(\varphi^{j}\right)_{j \in \llbracket 0,3 \rrbracket}\right)$ in $H^{1}\left(\Omega_{b}\right) \times \prod H^{1 / 2}\left(\Sigma_{a}^{j}\right)$ is solution of (29). Because, $\left(\varphi^{j}\right)_{j \in \llbracket 0,3 \rrbracket}$ satisfy the last two equations of (29), using the same arguments than in Section 3.1, we can construct unequivocally a function $p_{\text {ext }}$ in $H^{1}\left(\Omega \backslash \Omega_{a}\right)$ by

$$
\forall j \in \llbracket 0,3 \rrbracket,\left.\quad p_{\text {ext }}\right|_{\Omega_{a}^{j}}=P^{j}\left(\varphi^{j}\right) .
$$

which is solution of the homogeneous Helmholtz equation (4) with

$$
\forall j \in \llbracket 0,3 \rrbracket,\left.\quad p_{\text {ext }}\right|_{\Sigma_{a a}^{j}}=\left.\varphi^{j}\right|_{\Sigma_{a a}^{j}} .
$$

If $b>a$, as $p_{b}$ satisfies the first equation of (29) and by construction of $p_{\text {ext }}$, we deduce that $v=\left.p_{b}\right|_{\Omega_{b} \backslash \Omega_{a}}-\left.p_{\text {ext }}\right|_{\Omega_{b} \backslash \Omega_{a}}$ is solution in $H^{1}\left(\Omega_{b} \backslash \Omega_{a}\right)$ of

$$
-\triangle v-\omega_{\varepsilon}^{2} v=0
$$


and for all $j \in \llbracket 0,3 \rrbracket$,

$$
\begin{aligned}
& \left.v\right|_{\Sigma_{a a}^{j}}=\left.p_{b}\right|_{\Sigma_{a a}^{j}}-\left.\varphi^{j}\right|_{\Sigma_{a a}^{j}}=0, \quad \text { by }(64) \text { and the third equation of (29) } \\
& \left.\nabla v \cdot n^{j}\right|_{\Sigma_{b b}^{j}}=\left.\nabla p_{b} \cdot n^{j}\right|_{\Sigma_{b b}^{j}}-\Lambda^{j} \varphi^{j}=0 \text { by (63) and the second equation of (29), }
\end{aligned}
$$

where the last equality has to be understood in the $L^{2}$ sense because, by interior regularity arguments, $\Lambda^{j} \varphi^{j} \in L^{2}\left(\Sigma_{b b}^{j}\right)$ for $b>a$. We conclude that $p_{b}=p_{\text {ext }}$ in $\Omega_{b} \backslash \Omega_{a}$.

If $b=a$, using similar arguments, we show that

$$
\forall j,\left.\quad p_{b}\right|_{\Sigma_{a a}^{j}}=\left.p_{\text {ext }}\right|_{\Sigma_{a a}^{j}} \text { and }\left.\quad \nabla p_{b} \cdot n^{j}\right|_{\Sigma_{b b}^{j}}=\left.\nabla p_{\text {ext }} \cdot n^{j}\right|_{\Sigma_{b b}^{j}}
$$

which gives (by (21) for instance)

$$
\left.p_{b}\right|_{\Sigma_{a a}}=\left.p_{\text {ext }}\right|_{\Sigma_{a a}} \text { and }\left.\quad \nabla p_{b} \cdot n\right|_{\Sigma_{b b}}=\left.\nabla p_{\text {ext }} \cdot n\right|_{\Sigma_{b b}}
$$

In both cases, we can construct (unequivocally if $b>a$ ) a function $p$ in $H^{1}(\Omega)$ by

$$
\left.p\right|_{\Omega_{b}}=p_{b} \quad \text { and }\left.\quad p\right|_{\Omega \backslash \Omega_{b}}=\left.p_{\text {ext }}\right|_{\Omega \backslash \Omega_{b}}
$$

which is solution of (1) in the isotropic case.

Remark 4.1. As you can notice, in this proof, we do not use the expression of the solution of the halfspace problems or the expression of the operators. The same arguments can be used for the anisotropic case. That is why, Proposition 2.4 holds for the anisotropic case.

\subsection{Stability properties of Problem (29)}

Let us remind the reader that a variational formulation of (29) can only be derived for $b>a$. Indeed the difficulty for $b=a$ is that, we define piece by piece the normal trace of $p^{b}$ on $\Sigma_{a a}$ thanks to the restriction of $\Lambda \varphi^{j}$ on $\Sigma_{a a}^{j}$ which is in $H^{-1 / 2}\left(\Sigma_{a a}^{j}\right)$, the dual space of $\tilde{H}^{1 / 2}\left(\Sigma_{a a}^{j}\right)$, the set of functions of $H^{1 / 2}\left(\Sigma_{a a}^{j}\right)$ whose extension by 0 are in $H^{1 / 2}\left(\Sigma_{a a}\right)$. It seems then impossible to derive a variational formulation in that case. This is similar to the well known difficulty of the cross points in Domain Decomposition methods (14). However, this difficulty does not appear when $b>a$ because by interior regularity arguments, we know that the restriction of $\Lambda \varphi^{j}$ on $\Sigma_{b b}^{j}$ is at least 
in $L^{2}\left(\Sigma_{b b}^{j}\right)$. Introducing for all $j \varphi_{0}^{j}=\varphi^{j}-\left.p_{b}\right|_{\Sigma_{a a}^{j}}$ and using the notation introduced in (59), we can rewrite the variational formulation as follows

Find $\left(p_{b}, \Phi_{0}=\left(\varphi_{0}^{j}\right)_{j \in \llbracket 0,3 \rrbracket}\right) \in H^{1}\left(\Omega_{b}\right) \times V_{0}$ such that $\forall\left(q_{b}, \Psi_{0}\right) \in H^{1}\left(\Omega_{b}\right) \times V_{0}$

$$
\begin{array}{r}
\left(\left(\mathbb{S}-\omega_{\varepsilon}^{2} \mathbb{M}\right) p_{b}, q_{b}\right)_{\Omega_{b}}+((\mathbb{I}-\mathbb{L}) \Phi, \Psi)_{V_{0}}-(\mathbb{K} \Phi, \Psi)_{V_{0}}-\left(\mathbb{B} \gamma p_{b}, \Psi\right)_{V_{0}} \\
-\sum_{j=0}^{3}\left[\left(\Lambda^{j} \gamma^{j} p_{b}, q_{b}\right)_{\Sigma_{b b}^{j}}-\left(\Lambda^{j} \varphi_{0}^{j}, q_{b}\right)_{\Sigma_{b b}^{j}}\right]=\int_{\Omega_{b}} f \overline{q_{b}}
\end{array}
$$

where $V_{0}$ is defined in (19), the operator $\mathbb{S}$ is the stiffness operator, the operator $\mathbb{M}$ is the mass operator, the operator $\gamma\left(\right.$ resp. $\left.\gamma^{j}\right)$ is the trace operator from $H^{1}\left(\Omega_{b}\right)$ to $L^{2}\left(\Sigma_{a a}\right)$ (resp. from $H^{1}\left(\Omega_{b}\right)$ to $L^{2}\left(\Sigma_{a a}^{j}\right)$ ) and where as explained in Section 4.2, the norm of the operator $\mathbb{L} \in \mathcal{L}\left(V_{0}\right)$ is bounded by $1 / \sqrt{2}$ and the operator $\mathbb{K}$ is compact.

To show that this problem is of Fredholm type, it suffices to show the following property of the operator $\Lambda$.

Proposition 4.2. If $b>a$, for each $j \in\{0,1,2,3\}$, the operator $\Lambda^{j}$ defined in (36) is an operator from $L^{2}\left(\Sigma_{a}^{j}\right)$ to $L^{2}\left(\Sigma_{b b}^{j}\right)$ and is compact.

Proof: The proof can be done in two ways. The first way uses an abstract argument: it suffices to use the interior regularity property of the solution of the associated halfspace problem (8). Indeed, for any data $\varphi$ in $L^{2}\left(\Sigma_{a}^{j}\right)$, the solution of the associated halfspace problem (8) is $C^{\infty}$ far from the interface so in particular on $\Sigma_{b b}^{j}$ if $b>a$. The operator is then well defined from $L^{2}\left(\Sigma_{a}^{j}\right)$ to $L^{2}\left(\Sigma_{b b}^{j}\right)$. Compact embeddings results complete the proof.

The second way uses a more explicit argument: $\Lambda^{j}$ are isomorphic to $\Lambda$ as explained in (27) and $\Lambda$ is an Hilbert Schmidt operator from $L^{2}(\mathbb{R})$ to $L^{2}(-b, b)$ since the associated kernel given by

$$
k(y, \xi)=\imath \sqrt{\omega_{\varepsilon}^{2}-\xi^{2}} e^{\imath \sqrt{\omega_{\varepsilon}^{2}-\xi^{2}}(b-a)} e^{\imath \xi y} .
$$

is in $L^{2}((-b, b) \times \mathbb{R})$ if $b>a$. Indeed, we have

$$
\int_{\mathbb{R}} \int_{b}^{+\infty}|k(y, \xi)|^{2} d y d \xi \leq C \int_{\mathbb{R}}\left|\omega_{\varepsilon}^{2}-\xi^{2}\right| e^{-2 \operatorname{Im}\left(\sqrt{\omega_{\varepsilon}^{2}-\xi^{2}}\right)(b-a)}<+\infty .
$$


We can now conclude on the properties of the operators involved in the variational formulation (65). The two first terms define an operator which is coercive in $H^{1}\left(\Omega_{b}\right) \times V_{0}$. The other terms are compact operators in $H^{1}\left(\Omega_{b}\right) \times V_{0}$ since respectively: (1) $\mathbb{K}$ is compact in $V_{0}$ by Section $4.2,(2)$ the operator $\gamma$ (resp. the operators $\gamma^{j}$ ) is compact from $H^{1}\left(\Omega_{b}\right)$ to $V_{0}$ thanks to the compact embedding of $H^{1 / 2}\left(\Sigma_{a a}\right)$ in $L^{2}\left(\Sigma_{a a}\right)$ (resp. the compact embedding of $H^{1 / 2}\left(\Sigma_{a a}^{j}\right)$ in $L^{2}\left(\Sigma_{a a}^{j}\right)$ ) (3) thanks to Proposition 4.2 .

To show well-posedness of (65), it suffices then to show uniqueness of the solution. The difficulty has been already handled in Section 3.3. Indeed, similarly to Section 4.1, let us suppose that $\left(p_{b},\left(\varphi^{j}\right)_{j \in \llbracket 0,3 \rrbracket}\right)$ in $H^{1}\left(\Omega_{b}\right) \times \prod L^{2}\left(\Sigma_{a}^{j}\right)$ is solution of (29) with $f=0$. Using the well-posedness of Problem (17) with $g=\left.p_{b}\right|_{\Sigma_{a a}^{j}}$, it exists a unique solution $\left(\varphi_{j}\right)_{j \in \llbracket 0,3 \rrbracket}$ in $\prod L^{2}\left(\Sigma_{a}^{j}\right)$ solution of $(17)$, and this solution corresponds necessarily to the traces of the $H^{1}$ solution $p_{\text {ext }}$ of the exterior problem whose trace on $\partial \Omega_{a}$ is $g=\left.p_{b}\right|_{\Sigma_{a a}^{j}}$. The $\left(\varphi_{j}\right)_{j \in \llbracket 0,3 \rrbracket}$ are then in $\prod H^{1 / 2}\left(\Sigma_{a}^{j}\right)$ and it suffices then to conclude as in 3.3. As $f=0$, the unknowns $\left(p_{b},\left(\varphi^{j}\right)_{j \in \llbracket 0,3 \rrbracket}\right)$ vanish as well.

\section{Theoretical results associated to Case (3)}

The aim of this section is to prove Theorem 2.6 that states that Problem (37) is of Fredholm-type and it is well posed.

Comparing to the Case (2), the specificity is that the operators $D_{j \pm 1}^{j}$ and $\Lambda^{j}$ defined in (35) and (36) are a priori different the one from the others, in the sense that they cannot be identified to the same operators $D_{ \pm}$and $\Lambda$. In the isotropic case, the properties of the operators $D_{ \pm}$and $\Lambda$ given in Propositions $3.1,3.4$ and 4.2 were fundamental to show the stability properties of (31). In the anisotropic case, we then need to study for which conditions the same properties hold for the operators $D_{j \pm 1}^{j}$ and $\Lambda^{j}$.

Because of the presence of the overlap $(b>a)$, Proposition 4.2 holds even for the anisotropic case, and then for all $j \in \llbracket 0,3 \rrbracket$, the $\Lambda^{j}$ are compact operators.

It is less obvious to extend to the anisotropic case the results of Propositions 3.1 and 3.4. We remind the reader that for any $j$ and for any $\psi \in H^{1 / 2}\left(\Sigma_{a}^{j}\right)$, 
$P^{j}(\psi)$ denotes the unique solution in $H^{1}\left(\Omega_{a}^{j}\right)$ of the halfspace problem (32) which can be written in the variables $\left(x_{j}, y_{j}\right)$ introduced in (5)

$$
\mid \begin{array}{ll}
-\nabla_{\left(x_{j}, y_{j}\right)} \cdot\left(A_{0}^{j} \nabla_{\left(x_{j}, y_{j}\right)} P^{j}\right)-\omega_{\varepsilon}^{2} P^{j}=0 & \text { in } \Omega_{a}^{j} \\
P^{j}=\psi & \text { on } \Sigma_{a}^{j}
\end{array}
$$

with

$$
A_{0}^{0}=A_{0}^{2}=A_{0}:=\left(\begin{array}{ll}
c_{1} & c_{3} \\
c_{3} & c_{2}
\end{array}\right) \quad \text { and } \quad A_{0}^{1}=A_{0}^{3}=\left(\begin{array}{cc}
c_{2} & -c_{3} \\
-c_{3} & c_{1}
\end{array}\right)
$$

where we have supposed $c_{1}, c_{2}>0$ and $d:=c_{1} c_{2}-\left(c_{3}\right)^{2}>0$. Let us define for the sequel, the restriction operator $R_{I}^{j}$ of $L^{2}\left(\Sigma_{a}^{j}\right)$, for all $j \in \llbracket 0,3 \rrbracket$ and for all interval $I \subset \mathbb{R}$, defined by

$$
\forall \varphi \in L^{2}\left(\Sigma_{a}^{j}\right), \quad R_{I}^{j} \varphi\left(x_{j}=a, y_{j}\right)=\left\{\begin{array}{lll}
\varphi\left(x_{j}=a, y_{j}\right) & \text { for } & y_{j} \in I \\
0 & \text { for } & y_{j} \notin I
\end{array}\right.
$$

Let us focus for a moment on the problem $j=0$. By performing the change of variables (see Figure 6)

$$
X_{0}=\frac{x_{0}}{\sqrt{c_{1}}} \quad \text { and } \quad Y_{0}=\frac{\sqrt{c_{1}} y_{0}-c_{3} x_{0} / \sqrt{c_{1}}}{\sqrt{d}}
$$
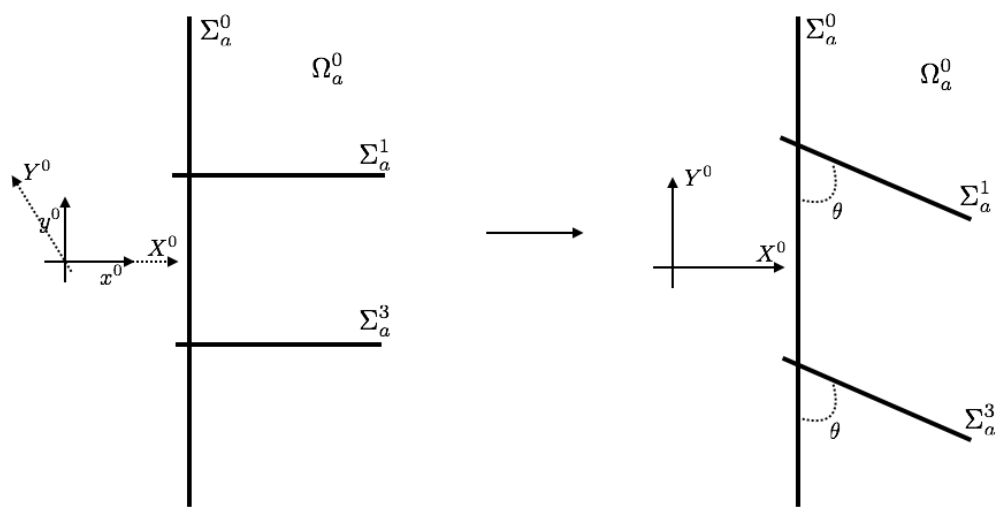

Figure 6: Change of variable $\left(x_{0}, y_{0}\right) \rightarrow\left(X_{0}, Y_{0}\right)$ 
we can show that the function $V^{0}(\Psi)\left(X_{0}, Y_{0}\right)=P^{0}(\psi)\left(x_{0}, y_{0}\right)$ is solution of the problem

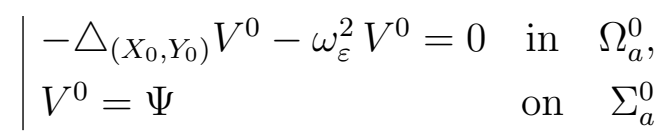

where $\Psi\left(Y_{0}\right)=\psi\left(\sqrt{d / c_{1}} Y_{0}+c_{3} a / c_{1}\right)$. Let us denote the lines

$$
\begin{gathered}
\Sigma_{a}^{1} \cap \Omega_{a}^{0}=\left\{\left(X_{0}, Y_{0}\right), X_{0} \geq \frac{a}{\sqrt{c_{1}}}, Y_{0}=\frac{\sqrt{c_{1}}}{\sqrt{d}} a-\frac{c_{3}}{\sqrt{d}} X_{0}\right\} \\
\Sigma_{a}^{3} \cap \Omega_{a}^{0}=\left\{\left(X_{0}, Y_{0}\right), X_{0} \geq \frac{a}{\sqrt{c_{1}}}, Y_{0}=-\frac{\sqrt{c_{1}}}{\sqrt{d}} a-\frac{c_{3}}{\sqrt{d}} X_{0}\right\} .
\end{gathered}
$$

Using the results of Propositions 3.1, 3.4 and Appendix A, we can deduce the following result on the operators $D_{1}^{0}$ and $D_{3}^{0}$.

Proposition 5.1. The operators $D_{1}^{0}$ and $D_{3}^{0}$ are continuous respectively from $L^{2}\left(\Sigma_{a}^{0}\right)$ to $L^{2}\left(\Sigma_{a}^{1} \cap \Omega_{a}^{0}\right)$ and from $L^{2}\left(\Sigma_{a}^{0}\right)$ to $L^{2}\left(\Sigma_{a}^{3} \cap \Omega_{a}^{0}\right)$. Moreover,

1. $D_{1}^{0} R_{(a,+\infty)}^{0}$ and $D_{3}^{0} R_{(-\infty,-a)}^{0}$ can be decomposed as

$$
\mid \begin{aligned}
& D_{1}^{0} R_{(a,+\infty)}^{0}=L_{1}^{0} R_{(a,+\infty)}^{0}+K_{1}^{0} \\
& D_{3}^{0} R_{(-\infty,-a)}^{0}=L_{3}^{0} R_{(-\infty,-a)}^{0}+K_{3}^{0}
\end{aligned}
$$

where $K_{1}^{0}$ (resp. $\left.K_{3}^{0}\right)$ is a compact operator from $L^{2}\left(\Sigma_{a}^{0}\right)$ to $L^{2}\left(\Sigma_{a}^{1} \cap \Omega_{a}^{0}\right)$ (resp. from $L^{2}\left(\Sigma_{a}^{0}\right)$ to $L^{2}\left(\Sigma_{a}^{1} \cap \Omega_{a}^{3}\right)$ ), $L_{1}^{0}$ (resp. $\left.L_{3}^{0}\right)$ is a continuous operator from $L^{2}\left(\Sigma_{a}^{0}\right)$ to $L^{2}\left(\Sigma_{a}^{1} \cap \Omega_{a}^{0}\right)$ (resp. $L^{2}\left(\Sigma_{a}^{0}\right)$ to $L^{2}\left(\Sigma_{a}^{1} \cap \Omega_{a}^{3}\right)$ ), and $L_{1}^{0}$ and $L_{3}^{0}$ satisfy for all $\varphi \in L^{2}\left(\Sigma_{a}^{0}\right)$

$$
\mid \begin{aligned}
& \left\|L_{1}^{0} R_{(a,+\infty)}^{0} \varphi\right\|_{L^{2}} \leq|\cos (\theta / 2)| \sqrt{\frac{c_{1}}{\sqrt{d}}}\left\|R_{(a,+\infty)}^{0} \varphi\right\|_{L^{2}\left(\Sigma_{a}^{0}\right)}, \\
& \left\|L_{3}^{0} R_{(-\infty,-a)}^{0} \varphi\right\|_{L^{2}} \leq|\sin (\theta / 2)| \sqrt{\frac{c_{1}}{\sqrt{d}}}\left\|R_{(-\infty,-a)}^{0} \varphi\right\|_{L^{2}\left(\Sigma_{a}^{0}\right)}
\end{aligned}
$$

where $\theta$ is defined by $\tan \theta=\sqrt{d} / c_{3}, d=c_{1} c_{2}-c_{3}^{2}$ if $c_{3} \neq 0$ and $\theta=\pi / 2$ if $c_{3}=0$.

2. $D_{1}^{0} R_{(-\infty,-a)}^{0}$ are $D_{3}^{0} R_{(a,+\infty)}^{0}$ are compact operators.

Proof: The continuity of the operators $D_{1}^{0}$ and $D_{3}^{0}$ is easy to deduce using Problem (69) in the new variables $\left(X_{0}, Y_{0}\right)$ and Appendix A.2. Similarly, 
using Appendix A.2 we have that the operators $D_{1}^{0} R_{(-\infty,-a)}^{0}$ and $D_{3}^{0} R_{(a,+\infty)}^{0}$ are compact operators.

Then, as in Section 3.2, the idea is to decompose $D_{1}^{0} R_{(a,+\infty)}^{0}$ and $D_{3}^{0} R_{(-\infty,-a)}^{0}$ as follows

$$
\begin{gathered}
D_{1}^{0} R_{(a,+\infty)}^{0}=R_{(a, b)}^{1} D_{1}^{0} R_{(a,+\infty)}^{0}+R_{(b,+\infty)}^{1} D_{1}^{0} R_{(a,+\infty)}^{0} \\
D_{3}^{0} R_{(-\infty,-a)}^{0}=R_{(a, b)}^{3} D_{3}^{0} R_{(-\infty,-a)}^{0}+R_{(b,+\infty)}^{3} D_{3}^{0} R_{(-\infty,-a)}^{0}
\end{gathered}
$$

Similarly to the proof of Lemma 3.2 , it is easy to see that $R_{(b,+\infty)}^{j} D_{j}^{0}$ with $b>a$ are compact operators for $j=1,3$.

To finish, using the same arguments than in the proof of Lemma 3.3 and Appendix A, we can show that

$R_{(a, b)}^{1} D_{1}^{0} R_{(a,+\infty)}^{0}=L_{1}^{0} R_{(a,+\infty)}^{0}+K_{1}^{0}, \quad$ and $\quad R_{(a, b)}^{3} D_{3}^{0} R_{(-\infty,-a)}^{0}=L_{3}^{0} R_{(-\infty,-a)}^{0}+K_{3}^{0}$

where 1) $K_{1}^{0}$ and $K_{3}^{0}$ are compact operators and 2) $L_{1}^{0}$ and $L_{3}^{0}$ are continuous operators which satisfy

$$
\forall \varphi \in L^{2}\left(\Sigma_{a}^{0}\right), \quad \mid \begin{aligned}
& \left\|L_{1}^{0} R_{(a,+\infty)}^{0} \varphi\right\|_{L^{2}} \leq|\cos (\theta / 2)| \sqrt{\frac{c_{1}}{\sqrt{d}}}\left\|R_{(a,+\infty)}^{0} \varphi\right\|_{L^{2}\left(\Sigma_{a}^{0}\right)}, \\
& \left\|L_{3}^{0} R_{(-\infty,-a)}^{0} \varphi\right\|_{L^{2}} \leq|\sin (\theta / 2)| \sqrt{\frac{c_{1}}{\sqrt{d}}}\left\|R_{(-\infty,-a)}^{0} \varphi\right\|_{L^{2}\left(\Sigma_{a}^{0}\right)}
\end{aligned}
$$

and $\theta$ is defined by $\tan \theta=\sqrt{d} / c_{3}$ if $c_{3} \neq 0$ and $\theta=\pi / 2$ if $c_{3}=0$. Indeed, using Appendix A, we have that for all $\psi \in L^{2}\left(\Sigma_{a}^{0}\right)$ and by denoting $\Psi\left(Y_{0}\right)=$ $R_{(a,+\infty)}^{0} \psi\left(y_{0}\right)$

$$
\int_{\Sigma_{a}^{j} \cap \Omega_{a}^{0}}\left|V^{0}(\Psi)\left(X_{0}, Y_{0}\right)\right|^{2} d X_{0} \leq(\cos (\theta / 2))^{2} \int_{\Sigma_{a}^{0}}\left|\Psi\left(Y_{0}\right)\right|^{2} d Y_{0}
$$

Finally, by performing the change of variables to go back to the variables $\left(x_{0}, y_{0}\right)$, we obtain the the estimate for $L_{1}^{0}$. Similar arguments give the bound for the norm of $L_{3}^{0}$.

Finally, extending these results to each halfspace, we get the following result.

Proposition 5.2. For all $j \in \mathbb{Z} / 4 \mathbb{Z}$, the operator $D_{j \pm 1}^{j}$ is continuous from $L^{2}\left(\Sigma_{a}^{j}\right)$ to $L^{2}\left(\Sigma_{a}^{j \pm 1} \cap \Omega_{a}^{j}\right)$. Moreover, 
1. $D_{j+1}^{j} R_{(a,+\infty)}^{j}$ and $D_{j-1}^{j} R_{(-\infty,-a)}^{j}$ can be decomposed as

$$
\mid \begin{aligned}
& D_{j+1}^{j} R_{(a,+\infty)}^{j}=L_{j+1}^{j} R_{(a,+\infty)}^{j}+K_{j+1}^{j} \\
& D_{j-1}^{j} R_{(-\infty,-a)}^{j}=L_{j-1}^{j} R_{(-\infty,-a)}^{j}+K_{j-1}^{j}
\end{aligned}
$$

where $K_{j \pm 1}^{j}$ are compact operators and $L_{j \pm 1}^{j}$ are continuous operators from $L^{2}\left(\Sigma_{a}^{j}\right)$ to $L^{2}\left(\Sigma_{a}^{j} \cap \Omega_{a}^{j \pm 1}\right)$ that satisfy for all $\varphi \in L^{2}\left(\Sigma_{a}^{j}\right)$

$$
\mid \begin{aligned}
& \left\|L_{j+1}^{j} R_{(a,+\infty)}^{j} \varphi\right\|_{L^{2}} \leq C_{j+1}^{j}\left\|R_{(a,+\infty)}^{j} \varphi\right\|_{L^{2}\left(\Sigma_{a}^{j}\right)} \\
& \left\|L_{j-1}^{j} R_{(-\infty,-a)}^{j} \varphi\right\|_{L^{2}} \leq C_{j-1}^{j}\left\|R_{(-\infty,-a)}^{j} \varphi\right\|_{L^{2}\left(\Sigma_{a}^{j}\right)}
\end{aligned}
$$

where

$$
C_{j+1}^{j}=\cos (\theta / 2) \sqrt{\frac{c_{1}}{\sqrt{d}}} \quad \text { and } \quad C_{j-1}^{j}=\sin (\theta / 2) \sqrt{\frac{c_{1}}{\sqrt{d}}} \quad \text { for } \quad j=0,2
$$

and

$$
C_{j+1}^{j}=\cos (\theta / 2) \sqrt{\frac{c_{2}}{\sqrt{d}}} \quad \text { and } \quad C_{j-1}^{j}=\sin (\theta / 2) \sqrt{\frac{c_{2}}{\sqrt{d}}} \quad \text { for } \quad j=1,3
$$

where $\theta$ is defined by $\tan \theta=\sqrt{d} / c_{3}, d=c_{1} c_{2}-c_{3}^{2}$ if $c_{3} \neq 0$ and $\theta=\pi / 2$ if $c_{3}=0$.

2. $D_{j+1}^{j} R_{(-\infty,-a)}^{j}$ and $D_{j-1}^{j} R_{(a,+\infty)}^{j}$ are compact operators.

Using the same arguments than for the proof of Theorem 2.5 detailed in Section 4.2 , we can show the first part of Theorem 2.6 concerning the stability property under the conditions (38) since

$$
C_{j \pm 1}^{j} \leq \max (|\cos (\theta / 2)|,|\sin (\theta / 2)|) \frac{\max \left(\sqrt{c_{1}}, \sqrt{c_{2}}\right)}{d^{1 / 4}}
$$

If the condition (38) is not satisfied, we can still show that the problem is of Fredholm type. Indeed, by Proposition 5.2, we can decompose $\mathbb{A}=\mathbb{I}-\mathbb{L}-\mathbb{K}$ as in (59) where $\mathbb{K}$ is compact and $\mathbb{L} \in \mathcal{L}\left(V_{0}\right)$ and

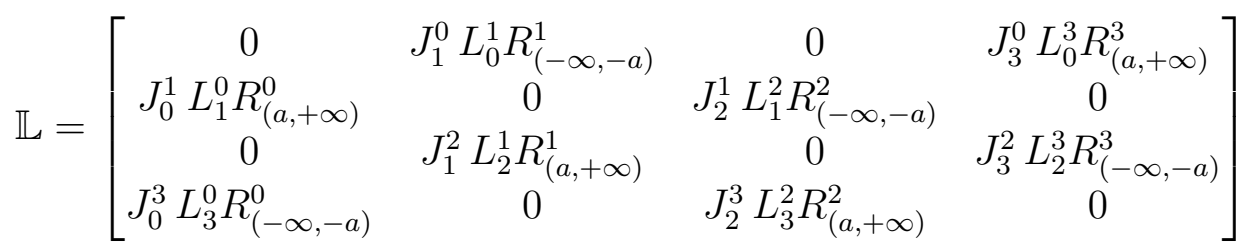


where $J_{j \pm 1}^{j}$ is the extension by 0 to the line $\Sigma_{a}^{j}$ of a function of $L^{2}\left(\Sigma_{a}^{j} \cap \Omega_{a}^{j \pm 1}\right)$. The difficulty here comes from the fact that the norm of $\mathbb{L}$ is, in general, not strictly less than 1 . Yet, we can still prove that $\mathbb{I}-\mathbb{L}$ is invertible thanks to the following result.

Proposition 5.3. The operator $\mathbb{L}^{2}$ is continuous from $V_{0}$ into itself and its norm is bounded by $1 / 2$.

Proof: Noticing that $R_{(a,+\infty)}^{j+1} J_{j}^{j+1}$ and $R_{(-\infty,-a)}^{j-1} J_{j}^{j-1}$ are equal to 0 , we deduce that $\mathbb{L}^{2}$ is a diagonal operator in the sense that

$$
\forall \Phi=\left(\varphi^{j}\right)_{j \in \llbracket 0,3 \rrbracket} \in \prod L^{2}\left(\Sigma_{a}^{j}\right), \quad\left(\mathbb{L}^{2} \Phi\right)^{j}=\mathbb{L}_{j j}^{2} \varphi^{j}
$$

where for all $j \in \mathbb{Z} / 4 \mathbb{Z}$

$\mathbb{L}_{j j}^{2}=J_{j+1}^{j} L_{j}^{j+1} R_{(-\infty,-a)}^{j+1} J_{j}^{j+1} L_{j+1}^{j} R_{(a,+\infty)}^{j}+J_{j-1}^{j} L_{j}^{j-1} R_{(a,+\infty)}^{j-1} J_{j}^{j-1} L_{j-1}^{j} R_{(-\infty,-a)}^{j}$.

Then, by Proposition 5.2, we get that for any $\varphi^{j} \in L^{2}\left(\Sigma_{a}^{j}\right)$

$$
\begin{aligned}
\left\|J_{j+1}^{j} L_{j}^{j+1} R_{(-\infty,-a)}^{j+1} J_{j}^{j+1} L_{j+1}^{j} R_{(a,+\infty)}^{j} \varphi^{j}\right\| & \leq|\sin (\theta / 2) \cos (\theta / 2)| \frac{\sqrt{c_{1} c_{2}}}{\sqrt{d}}\left\|R_{(a,+\infty)}^{j} \varphi^{j}\right\| \\
\leq & \frac{1}{2}|\sin (\theta)| \frac{\sqrt{c_{1} c_{2}}}{\sqrt{d}}\left\|R_{(a,+\infty)}^{j} \varphi^{j}\right\| . \quad(72)
\end{aligned}
$$

If $c_{3} \neq 0$, we recall that $\theta=\arctan \left(\sqrt{d} / c_{3}\right)$ and $d=c_{1} c_{2}-\left(c_{3}\right)^{2}$ so that

$$
\sin (\theta)=\frac{\sqrt{d} / c_{3}}{\sqrt{\left(\sqrt{d} / c_{3}\right)^{2}+1}}=\frac{\sqrt{d}}{\sqrt{c_{1} c_{2}}}
$$

Then, it comes in $(72)$

$$
\left\|J_{j+1}^{j} L_{j}^{j+1} R_{(-\infty,-a)}^{j+1} J_{j}^{j+1} L_{j+1}^{j} R_{(a,+\infty)}^{j} \varphi^{j}\right\| \leq \frac{1}{2}\left\|R_{(a,+\infty)}^{j} \varphi^{j}\right\| .
$$

We prove exactly the same result in the particular case $c_{3}=0$. In the same manner, we get

$$
\left\|J_{j-1}^{j} L_{j}^{j-1} R_{(a,+\infty)}^{j-1} J_{j}^{j-1} L_{j-1}^{j} R_{(-\infty,-a)}^{j} \varphi^{j}\right\| \leq \frac{1}{2}\left\|R_{(-\infty,-a)}^{j} \varphi^{j}\right\| .
$$


By definition of the extension operators $J_{j \pm 1}^{j}$, we have

$$
\begin{aligned}
\left\|\mathbb{L}_{j j}^{2} \varphi^{j}\right\|^{2}=\left\|L_{j}^{j+1} R_{(-\infty,-a)}^{j+1} J_{j}^{j+1} L_{j+1}^{j} R_{(a,+\infty)}^{j} \varphi^{j}\right\|^{2} & \\
& +\left\|L_{j}^{j-1} R_{(a,+\infty)}^{j-1} J_{j}^{j-1} L_{j-1}^{j} R_{(-\infty,-a)}^{j} \varphi^{j}\right\|^{2}
\end{aligned}
$$

which gives thanks to the previous inequalities

$$
\left\|\left(\mathbb{L}^{2}\right)_{j j} \varphi^{j}\right\|^{2} \leq \frac{1}{4}\left\|\varphi^{j}\right\|^{2} .
$$

We conclude easily that the norm of $\mathbb{L}^{2}$ is bounded by $1 / 2$.

With this result, we easily deduce that $\mathbb{I}-\mathbb{L}^{2}$ is invertible. Writing $\mathbb{I}-\mathbb{L}^{2}=$ $(\mathbb{I}-\mathbb{L})(\mathbb{I}+\mathbb{L})$, we have $\operatorname{Im}\left(\mathbb{I}-\mathbb{L}^{2}\right) \subset \operatorname{Im}(\mathbb{I}-\mathbb{L})$ so $\mathbb{I}-\mathbb{L}$ is surjective. Similarly, since $\mathbb{I}-\mathbb{L}^{2}=(\mathbb{I}+\mathbb{L})(\mathbb{I}-\mathbb{L})$, we have $\operatorname{Ker}(\mathbb{I}-\mathbb{L}) \subset \operatorname{Ker}\left(\mathbb{I}-\mathbb{L}^{2}\right)$, so $\mathbb{I}-\mathbb{L}$ is injective. Therefore $\mathbb{I}-\mathbb{L}$ is also invertible (but not necessarily coercive). This shows that Freholm alternative holds for Problem (37).

Remark 5.4. Let us emphasize than in the general anisotropic case, the operator involved in Problem (37) cannot be decomposed as the sum of a coercive operator and a compact one but only as the sum of an invertible one and a compact one. The well posedness is ensured by the uniqueness which is showed in the sequel. The consequence of this weaker result is that a priori, the numerical analysis cannot be done in the general case. However, Problem (37) can be adapted to recover the stability property. Indeed, we can show that a solution of (37) is also solution of a similar problem where the $\mathbb{A}$ is replaced by an operator $\tilde{\mathbb{A}}=\mathbb{I}-(\mathbb{I}-\mathbb{A})^{2}$ and the operator $\mathbb{B}$ by $\tilde{\mathbb{B}}=(2 \mathbb{I}-\mathbb{A}) \mathbb{B}$.

Concerning the well-posedness of Problem (37), as explained in Section 4.2, the only difficulty concerns the extension of the well-posedness of (20), whose proof is detailed in Section 3.3, to the anisotropic case. In order to extend the proof, it suffices to answer the two intrincate following questions.

1. Does the function $V \in L^{2}\left(Q_{a}^{01}\right)$ where $Q_{a}^{01}=\Omega_{a}^{0} \cap \Omega_{a}^{1}$ which is solution in the distributional sense of

$$
-\nabla \cdot\left(A_{0} \nabla V\right)-\omega_{\varepsilon}^{2} V=0, \quad \text { in } \quad Q_{a}^{01}
$$

and whose "trace" on the boundary of the quarter plane, vanishes in the sense of

$$
\forall u \in H^{2}\left(Q_{a}^{01}\right) \cap H_{0}^{1}\left(Q_{a}^{01}\right), \quad \int_{Q_{a}^{01}} \nabla \cdot\left(A_{0} \nabla V\right) u=\int_{Q_{a}^{01}} V \nabla \cdot\left(A_{0} \nabla u\right)
$$


vanish? By using the same change of variables than previously, this function $V$ is linked to the function satisfying same kind of equations but in the isotropic case $\left(A_{0}=\mathbb{I}\right)$ and in a sector of angle $\pi-\theta$ where the angle $\theta$ is defined in Proposition 5.2 (see figure 6). As $\theta \in(0, \pi)$, the sector is convex and using similar arguments than in Section 3.3, we can conclude that $V=0$.

2. Does the function $p \in L^{2}\left(\Omega \backslash \Omega_{a}\right)$ whose trace on each line $\Sigma_{a}^{j}$ is in $L^{2}$, which is solution in the distributional sense of

$$
-\nabla \cdot\left(A_{0} \nabla p\right)-\omega_{\varepsilon}^{2} p=0, \quad \text { in } \quad \Omega \backslash \Omega_{a}
$$

and whose trace vanishes on $\partial \Omega_{a}$ in the sense that

$\forall u \in H^{2}\left(\Omega \backslash \Omega_{a}\right) \cap H_{0}^{1}\left(\Omega \backslash \Omega_{a}\right), \quad \int_{\Omega \backslash \Omega_{a}} \nabla \cdot\left(A_{0} \nabla p\right) u=\int_{\Omega \backslash \Omega_{a}} p \nabla \cdot\left(A_{0} \nabla u\right)$

vanish? Again by the same change of variables, this function $p$ is linked to the function $\tilde{p}$ satisfying the same kind of equations but in the isotropic case and outside a parallelogram. The complementary of the parallelogram is not convex but as in Section 3.3, the function $\tilde{p}$ is in $H^{1}$ up to a linear combination of singular functions. We have then the same decomposition for the function $p$, but since the trace of $p$ on each line $\Sigma_{a}^{j}$ is in $L^{2}$, we can conclude that $p$ is in $H^{1}$, then $p=0$.

\section{Some extensions}

\section{$3 D$ configurations.}

The method extends straightforwardly to problems similar to (1) with $\Omega \subset$ $\mathbb{R}^{3}$. The lines $\Sigma_{a}^{j}$ are replaced by infinite planes and half-planes are replaced by half-spaces where analytical representation are derived using the 2D Fourier Transform. All theoretical results remain true. The method has not been yet implemented but we anticipate numerical difficulties linked to the presence of dense and large blocks in the matrix to invert. This could require appropriate compression strategies.

\section{Multi-scattering problems.}

In some cases, enclosing all the heterogeneities and obstacles in one convex polygon can be costly. For instance, in the case of two remote heterogeneities/obstacles, a more relevant formulation can be derived by introducing two separated rectangles (or more general polygons if necessary), one for 
each heterogeneity/obstacle. There will be 2 volumic unknowns, and 8 traces unknowns. The system of coupled equations will be essentially the same with additional coupling terms representing the coupling between the two obstacles. These coupling terms being "compact", the stability and well-posedness results can be proven with similar arguments.

\section{Scattering problems.}

Our main motivation is to apply our method to scattering problems which make sense in the case without dissipation. We refer the reader to the next section dedicated to the theoretical difficulties raised in the non dissipative case. Besides, our method extends directly to the scattering problems if the unknown is the diffracted field. An alternative could be to keep the total field for the volumic unknown and use the diffracted fields for the trace unknowns.

\section{The non dissipative case : Open questions but encouraging nu- merical results}

Up to now, we have considered the dissipative problem (4) with $\operatorname{Im}\left(\omega_{\varepsilon}\right)>0$. But we claim that in practice, our method also works in the non dissipative case $\operatorname{Im}(\omega)=0$ (See Figure 7). It is quite easy to extend the discrete formulation to this case, selecting carefully the outgoing solution of the halfspace problem. The price to pay in order to get accurate results is then to use a refined discretization in the Fourier variable $\xi$. Indeed, the solution decaying much more slowly than in the dissipative case (the solution in the

non-dissipative isotropic case behaves like $\frac{e^{i \omega r}}{\sqrt{r}}$ at infinity, while the solution in the dissipative case decays exponentially), its Fourier transform is less regular. 


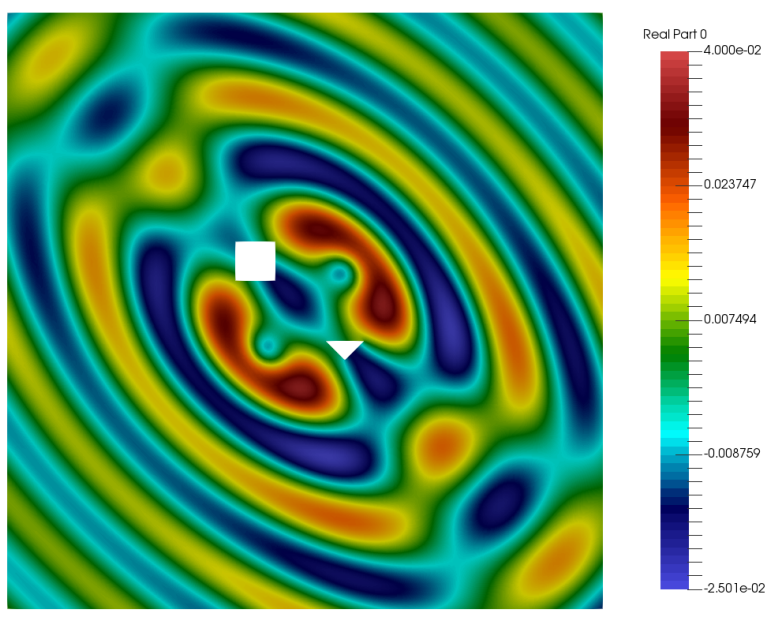

Figure 7: The solution in an anisotropic non dissipative case with $\omega=10$

The main difficulty lies at the theoretical level: the extension of the theoretical results to the non-dissipative case still raises many open questions, that we briefly discuss below.

Let us consider to fix ideas the simplest case of the (isotropic non-dissipative) Helmholtz equation. It is well-known that the well-posedness of the associated boundary value problem is ensured if one imposes to the solution to be "outgoing", which means for instance that the solution satisfies the Sommerfeld radiation condition at infinity. Our objective is to reformulate this problem using our halfspace matching formulation. The first difficulty is that the traces $\varphi^{j}$ of the solution are no longer in $L^{2}$ (again, they behave like $\left.\frac{e^{i \omega r}}{\sqrt{r}}\right)$. An appropriate functional framework has been introduced in $(9 ; 10)$, where the space $H^{1 / 2}(\mathbb{R})$, convenient for the dissipative case, is replaced by the space $V(\omega ; \mathbb{R})$ of the functions $\varphi$ such that $\left|\xi^{2}-\omega^{2}\right|^{1 / 4} \hat{\varphi} \in L^{2}(\mathbb{R})$. The functions of $V(\omega ; \mathbb{R})$ are locally in $H^{1 / 2}$, but they may not belong to $L^{2}(\mathbb{R})$, due to their behavior at infinity. Using this framework we can state the following result: 
Let $g \in H^{1 / 2}\left(\Sigma_{a a}\right)$. If $p$ is the outgoing solution of

$$
\mid \begin{array}{lll}
-\Delta p-\omega^{2} p=0 & \text { in } & \Omega=\mathbb{R}^{2} \backslash \Omega_{a} \\
p=g & \text { on } & \Sigma_{a a}
\end{array}
$$

then $\left\{\varphi_{a}^{j}, j \in\{0,1,2,3\}\right\}$ is solution of (16) in the space $\prod_{j=0}^{3} V\left(\omega ; \Sigma_{a}^{j}\right)$ (with obvious notations). But we are not able to prove the converse statement corresponding to the second part of Proposition 2.1 (even if we conjecture that it is true). Indeed, we did not succeed in proving that the different halfspace representations of the solution match in the quarters of planes. For instance, we are not able to prove like in paragraph 3.1 that $V=P^{0}\left(\varphi^{0}\right)-P^{1}\left(\varphi^{1}\right)$ vanishes in $\Omega_{a}^{0} \cap \Omega_{a}^{1}$. The reason is that we cannot prove that $V$ is outgoing (in the sense of Sommerfeld radiation condition at infinity), since we just know that $P^{0}\left(\varphi^{0}\right)$ is propagating in the direction of positive $x$, while $P^{1}\left(\varphi^{1}\right)$ is propagating in the direction of positive $y$.

Another difficulty arises when we try to extend stability results like Theorem 2.2 to the non-dissipative case. All the theory has been done with traces in $L^{2}$, which is no longer the appropriate space. An idea could be to introduce the space $L(\omega ; \mathbb{R})$ of the functions $\varphi$ such that $\frac{\left|\xi^{2}-\omega^{2}\right|^{1 / 4}}{\left|\xi^{2}+\omega^{2}\right|^{1 / 4}} \hat{\varphi} \in L^{2}(\mathbb{R})$, because the functions of $L(\omega ; \mathbb{R})$ are locally in $L^{2}$. But it is far from obvious to prove the different properties of the integral operators in this space. Finally, proving uniqueness will be of course much more intricate in the non-dissipative case than in the dissipative case, where we mainly used the coerciveness in $H^{1}$. A first step has been done by proving a related uniqueness result: in (7), overlapping halfspace representations are used to prove the absence of trapped modes (i.e. $L^{2}$ solutions of the homogeneous non-dissipative equation) under very weak hypotheses.

Les us emphasize that our approach seems to be well-suited to formulate a large class of problems, for which no equivalent of the Sommerfeld radiation condition is available. This is typically the case of anisotropic elastic media. For these problems, we aim at using our halfspace formulation, not only to solve numerically the problem, but also to define the notion of outgoing solution and to prove existence and uniqueness of this outgoing solution. This has been done for instance in (8) using a formulation with non-overlapping halfspace representations for the junction of open waveguides. 


\section{Appendix A. The case of a general convex polygon}

In Case (1), we have considered for now only the case where the obstacle $\partial \Omega$ is a square but the method can be extended for any convex polygon. There will be as many trace unknowns as edges of the polygon. This suggests that for Case (2) and Case (3), the domain $\Omega_{a}$ can be any convex polygon strictly included in $\Omega_{b}$ (i.e. whose shape can be chosen independently).

To illustrate this extension, we consider in this appendix the Case (1) when $\partial \Omega$ is a triangle (see Figure A.8). This situation is particularly of interest since in that case, only 3 trace unknowns are involved.

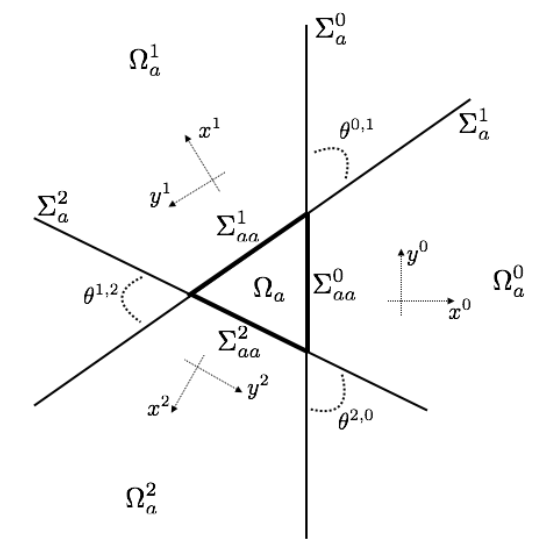

Figure A.8: $\Omega_{a}$ is a triangle and some associated notations

Let $\Omega=\mathbb{R}^{2} \backslash \Omega_{a}, g \in H^{1 / 2}(\partial \Omega)$ and find the unique solution $p \in H^{1}(\Omega)$ of

$$
\mid \begin{array}{ll}
-\Delta p-\omega_{\varepsilon}^{2} p=0 & \text { in } \Omega, \\
p=g & \text { on } \partial \Omega
\end{array}
$$

The domain $\Omega$ can be split into 3 half-planes $\Omega_{a}^{j}$ that lie on the 3 edges of the triangle $\Omega_{a}$. To define these 3 half-planes, we will use the following local coordinates

$$
\forall j \in \mathbb{Z} / 2 \mathbb{Z}, \quad\left[\begin{array}{l}
x^{j+1} \\
y^{j+1}
\end{array}\right]=\left[\begin{array}{cc}
-\cos \left(\theta^{j, j+1}\right) & \sin \left(\theta^{j, j+1}\right) \\
-\sin \left(\theta^{j, j+1}\right) & -\cos \left(\theta^{j, j+1}\right)
\end{array}\right]\left[\begin{array}{l}
x^{j} \\
y^{j}
\end{array}\right],
$$

where $\theta^{j, j+1} \in(0, \pi)$ is represented in Figure A.8 and $l^{j}$ is the distance of the origin to the lines $\Sigma_{a}^{j}$, and define the half-planes as follows:

$\Omega_{a}^{j}=\left\{x^{j} \geq l^{j}\right\} \times\left\{y^{j} \in \mathbb{R}\right\} \quad \Sigma_{a}^{j} \equiv \partial \Omega_{a}^{j}:=\left\{x^{j}=l^{j}\right\} \times\left\{y^{j} \in \mathbb{R}\right\} \quad \forall j \in \llbracket 0,2 \rrbracket$. 
Finally, we denote as before

$$
\Sigma_{a a}=\partial \Omega_{a} \quad \text { and } \quad \Sigma_{a a}^{j}=\Sigma_{a a} \cap \Sigma_{a}^{j} .
$$

These notations are summarized on Figure A.8. For each $j$, the expression (9) of the solution of the associated halfspace problem (8) is the same but the expression of the operators $D_{j \pm 1}^{j}$ defined in (14) is different. In particular, they are not necessarily isomorphic to the same operators $D_{ \pm}$since the angle between the $\Sigma_{a}^{j}$ 's are different. They are given by for all $\psi \in H^{1 / 2}\left(\Sigma_{a}^{j}\right)$

$$
D_{j \pm 1}^{j} \psi(t)=\frac{1}{\sqrt{2 \pi}} \int_{\mathbb{R}} \widehat{\psi}(\xi) e^{\imath \sqrt{\omega_{\varepsilon}^{2}-\xi^{2}} t \sin \left(\theta^{j, j \pm 1}\right)} e^{ \pm \imath \xi\left(a^{j}+t \cos \left(\theta^{j, j \pm 1}\right)\right)} d \xi, \quad \text { for } t \geq 0 .
$$

where $2 a^{j}=\left|\Sigma_{a a}^{j}\right|$ and $\theta^{j, j \pm 1}=\theta^{j \pm 1, j}$.

Using the same approach than in Section 2.1, we introduce the following problem

$$
\forall j \in \mathbb{Z} / 2 \mathbb{Z}, \quad \varphi^{j}\left(y^{j}\right)=\mid \begin{array}{ll}
D_{j}^{j-1} \varphi^{j-1}\left(-\left(y^{j}+a^{j}\right)\right) & \text { for } y^{j} \in\left(-\infty,-a^{j}\right) \\
D_{j}^{j+1} \varphi^{j+1}\left(y^{j}-a^{j}\right) & \text { for } y^{j} \in\left(a^{j},+\infty\right) \\
g\left(y^{j}\right) & \text { for } y^{j} \in\left(-a^{j}, a^{j}\right) .
\end{array}
$$

We have the equivalent of Theorem 2.2 for the triangle case.

Theorem Appendix A.1. (1) Let $g \in L^{2}\left(\Sigma_{a a}\right)$, the problem

$$
\text { Look for }\left(\varphi^{j}\right)_{j \in \llbracket 0,2 \rrbracket} \in \prod_{j=0}^{3} L^{2}\left(\Sigma_{a}^{j}\right) \quad \text { solution of (A.4) }
$$

is of Fredholm type.

(2) Problem (A.5) is well posed in $L^{2}$.

The stability property is a consequence of the following result.

Proposition Appendix A.2. The operator $D_{j \pm 1}^{j}$ is continuous from $L^{2}\left(\Sigma_{a}^{j}\right)$ to $L^{2}\left(\Sigma_{a}^{j \pm 1} \cap \Omega_{a}^{j}\right)$. Moreover, 
1. $D_{j+1}^{j} R_{\left(a^{j},+\infty\right)}^{j}$ and $D_{j-1}^{j} R_{\left(-\infty,-a^{j}\right)}^{j}$ can be decomposed as

$$
\mid \begin{aligned}
& D_{j+1}^{j} R_{\left(a^{j},+\infty\right)}^{j}=L_{j+1}^{j} R_{\left(a^{j},+\infty\right)}^{j}+K_{j+1}^{j} \\
& D_{j-1}^{j} R_{\left(-\infty,-a^{j}\right)}^{j}=L_{j-1}^{j} R_{\left(-\infty,-a^{j}\right)}^{j}+K_{j-1}^{j}
\end{aligned}
$$

where $K_{j \pm 1}^{j}$ are compact operators and $L_{j \pm 1}^{j}$ are continuous operators from $L^{2}\left(\Sigma_{a}^{j}\right)$ to $L^{2}\left(\Sigma_{a}^{j} \cap \Omega_{a}^{j \pm 1}\right)$ that satisfy for all $\varphi$ in $L^{2}\left(\Sigma_{a}^{j}\right)$

$$
\mid \begin{aligned}
& \left\|L_{j+1}^{j} R_{\left(a^{j},+\infty\right)}^{j} \varphi\right\|_{L^{2}} \leq \cos \left(\theta^{j, j+1} / 2\right)\left\|R_{\left(a^{j},+\infty\right)}^{j} \varphi\right\|_{L^{2}} \\
& \left\|L_{j-1}^{j} R_{\left(-\infty,-a^{j}\right)}^{j} \varphi\right\|_{L^{2}} \leq \sin \left(\theta^{j, j-1} / 2\right)\left\|R_{\left(-\infty,-a^{j}\right)}^{j} \varphi\right\|_{L^{2}}
\end{aligned}
$$

2. $D_{j+1}^{j} R_{\left(-\infty,-a^{j}\right)}^{j}$ and $D_{j-1}^{j} R_{\left(a^{j},+\infty\right)}^{j}$ are compact operators.

Proof: The proof will be done only for $D_{1}^{0}$ and it can be extended to the other operators easily. We use the same steps for the proof as for the one described in Section 3.2. For the decomposition of $D_{1}^{0}$, it suffices to use a restriction operator, as in (45), which allows us to separate the behavior of the operator near the intersection of the lines $\Sigma_{a}^{0}$ and $\Sigma_{a}^{1}$ and far from this intersection. As in Lemma 3.2, it is easy to show that the part far from the intersection $R_{\left(-\infty,-b^{1}\right)}^{1} D_{1}^{0}$ is compact from $L^{2}\left(\Sigma_{a}^{0}\right)$ to $L^{2}\left(\Sigma_{a}^{1} \cap \Omega_{a}^{0}\right)$, for $b^{1}>a^{1}$. For the part near the intersection $R_{\left(-b^{1},-a^{1}\right)}^{1} D_{1}^{0}$, we study as in the proof of Lemma 3.3, the norm of

$$
L_{1}^{0} \varphi=\left.v(\varphi)\right|_{\theta=\theta^{0,1}}
$$

in $L^{2}\left(\mathbb{R}^{+}\right)$, where $v(\varphi)$ is solution of $(47)$ and where the polar coordinates $(r, \theta)$ are defined in (48). Using the same reasoning as in the proof of Lemma 3.3 , we have that

$$
\begin{aligned}
\left\|\left.v(\varphi)\right|_{\theta=\theta^{0,1}}\right\|_{L^{2}\left(\mathbb{R}^{+}\right)} \leq & \sup _{-1 / 2+\imath \mathbb{R}}\left|A\left(\lambda, \pi-\theta^{0,1}\right)\right|\|\varphi\|_{L^{2}\left(-\infty, a^{0}\right)} \\
& +\sup _{-1 / 2+\imath \mathbb{R}}\left|A\left(\lambda, \theta^{0,1}\right)\right|\|\varphi\|_{L^{2}\left(a^{0},+\infty\right)}
\end{aligned}
$$

where $A$ is defined in (55). Since

$$
\sup _{-1 / 2+\imath \mathbb{R}}\left|A\left(\lambda, \theta^{0,1}\right)\right|=\sin \left(\theta^{0,1} / 2\right),
$$

we can conclude that the operator $L_{1}^{0}$ is continuous, and also that

$$
\left\|L_{1}^{0} R_{\left(a^{0},+\infty\right)}^{0}\right\| \leq \sin \left(\theta^{0,1} / 2\right) .
$$


Finally, to prove the last point, $D_{1}^{0} R_{\left(-\infty,-a^{0}\right)}^{0}$ is compact, we use the same arguments as in the proof of Lemma 3.3.

To show stability, it suffices then to use exactly the same arguments than the ones detailed in Section 4.2. To show well posedness, the reasoning of 4.2 can be also adapted to that case.

\section{References}

[1] T. Apel, S. Nicaise, and J. Pfefferer. A dual singular complement method for the numerical solution of the poisson equation with $l 2$ boundary data in non-convex domains. arXiv preprint arXiv:1505.00414, 2015.

[2] T. Apel, S. Nicaise, and J. Pfefferer. Discretization of the poisson equation with non-smooth data and emphasis on non-convex domains. Numerical Methods for Partial Differential Equations, 32(5):1433-1454, 2016.

[3] E. Bécache, S. Fauqueux, and P. Joly. Stability of perfectly matched layers, group velocities and anisotropic waves. Journal of Computational Physics, 188(2):399-433, 2003.

[4] C. Boller and N. Meyendorf. State-of-the-art in structural health monitoring for aeronautics. In Proceedings of the International Symposium on NDT in Aerospace, 2008.

[5] M. Bonnet. A modified volume integral equation for anisotropic elastic or conducting inhomogeneities: Unconditional solvability by neumann series. Journal of Integral Equations and Applications, 29(2):271-295, 2017.

[6] A.-S. Bonnet-Ben Dhia, C. Chambeyron, and G. Legendre. On the use of perfectly matched layers in the presence of long or backward propagating guided elastic waves. Wave Motion, 51(2):266-283, 2014.

[7] A.-S. Bonnet-Ben Dhia, S. Fliss, C. Hazard, and A. Tonnoir. A rellich type theorem for the helmholtz equation in a conical domain. Comptes Rendus Mathematique, 354(1):27-32, 2016. 
[8] A.-S. Bonnet-Ben Dhia, B. Goursaud, and C. Hazard. Mathematical analysis of the junction of two acoustic open waveguides. SIAM Journal on Applied Mathematics, 71(6):2048-2071, 2011.

[9] A.-S. Bonnet-Ben Dhia and A. Tillequin. A generalized mode matching method for scattering problems with unbounded obstacles. Journal of Computational Acoustics, 9(04):1611-1631, 2001.

[10] A.-S. Bonnet-Ben Dhia and A. Tillequin. A limiting absorption principle for scattering problems with unbounded obstacles. Mathematical methods in the applied sciences, 24(14):1089-1111, 2001.

[11] M. Dryja and O. B. Widlund. Domain decomposition algorithms with small overlap. SIAM Journal on Scientific Computing, 15(3):604-620, 1994.

[12] S. Fliss. Étude mathématique et numérique de la propagation des ondes dans des milieux périodiques localement perturbés. $\mathrm{PhD}$ thesis, $\mathrm{PhD}$ thesis, Ecole Polytechnique, 112009.

[13] S. Fliss and P. Joly. Exact boundary conditions for time-harmonic wave propagation in locally perturbed periodic media. Applied Numerical Mathematics, 59(9):2155-2178, 2009.

[14] M. J. Gander and K. Santugini. Cross-points in domain decomposition methods with a finite element discretization. Electronic Transactions on Numerical Analysis, 45:219-240, 2016.

[15] D. Givoli. Numerical Methods for Problems in Infinite Domains. Elsevier Science Limited, 1992.

[16] D. Givoli and J. B. Keller. Non-reflecting boundary conditions for elastic waves. Wave motion, 12(3):261-279, 1990.

[17] P. Grisvard. Elliptic problems in nonsmooth domains, volume 69. SIAM, 2011.

[18] M. Halla, T. Hohage, L. Nannen, and J. Schöberl. Hardy space infinite elements for time harmonic wave equations with phase and group velocities of different signs. Numerische Mathematik, 133(1):103-139, 2016. 
[19] M. Halla and L. Nannen. Hardy space infinite elements for timeharmonic two-dimensional elastic waveguide problems. Wave Motion, 59:94-110, 2015.

[20] V. A. Kozlov, V.G. Mazia, and J. Rossmann. Elliptic boundary value problems in domains with point singularities, volume 52. American Mathematical Soc., 1997.

[21] J.-L. Lions and E. Magenes. Problèmes aux limites non homogènes et applications. Vol. 1. Travaux et Recherches Mathématiques, No. 17. Dunod, Paris, 1968.

[22] Pierre-Louis Lions. On the schwarz alternating method. i. In First international symposium on domain decomposition methods for partial differential equations, pages 1-42. Paris, France, 1988.

[23] E. A. Skelton, S. D.M. Adams, and R. V. Craster. Guided elastic waves and perfectly matched layers. Wave motion, 44(7):573-592, 2007.

[24] A. Tonnoir. Conditions transparentes pour la diffraction d'ondes en milieu élastique anisotrope. Thèse de l'Ecole Polytechnique, 2015.

[25] A. Toselli and O. B. Widlund. Domain decomposition methods: algorithms and theory, volume 34. Springer, 2005.

[26] C.-Y. Wang and J.D. Achenbach. Three-dimensional time-harmonic elastodynamic green's functions for anisotropic solids. In Proceedings of the Royal Society of London A: Mathematical, Physical and Engineering Sciences, volume 449, pages 441-458. The Royal Society, 1995. 